\title{
SEQUENTIAL STAR FORMATION IN RCW 34: A SPECTROSCOPIC CENSUS OF THE STELLAR CONTENT OF HIGH-MASS STAR-FORMING REGIONS*
}

\author{
A. BiK ${ }^{1}$, E. Puga ${ }^{2,3}$, L.B.F.M. Waters ${ }^{3,4}$, M. Horrobin ${ }^{4,5}$, Th. Henning ${ }^{1}$, T. VAsYunina ${ }^{1}$, H. Beuther ${ }^{1}$, H. LinZ ${ }^{1}$, L. KAPER ${ }^{4}$, \\ M. van den AnCKer ${ }^{6}$, A. Lenorzer ${ }^{7}$, E. Churchwell ${ }^{8}$, S. Kurtz ${ }^{9}$, M. B. N. Kouwenhoven ${ }^{10,11}$, A. Stolte ${ }^{5}$, \\ A. De Koter ${ }^{4,12}$, W. F. Thi ${ }^{13}$, F. Comerón ${ }^{6}$, And CH. Waelkens $^{3}$ \\ ${ }^{1}$ Max-Planck-Institut für Astronomie, Königstuhl 17, 69117 Heidelberg, Germany \\ ${ }^{2}$ Centro de Astrobiología (CSIC-INTA), 28850 Torrejón de Ardoz, Madrid, Spain \\ ${ }^{3}$ Instituut voor Sterrenkunde, Celestijnenlaan 200D, B-3001 Leuven, Belgium \\ ${ }^{4}$ Sterrenkundig Instituut “Anton Pannekoek," University of Amsterdam, Science Park 904, 1098 XH Amsterdam, The Netherlands \\ ${ }^{5}$ I.Physikalisches Institut, Universität zu Köln, 50937 Köln, Germany \\ ${ }^{6}$ European Southern Observatory, Karl-Schwarzschild Strasse 2, Garching-bei-München, D-85748, Germany \\ ${ }^{7}$ Instituto Astrofísica de Canarias, Vía Láctea s/n, E-38200 La Laguna, Spain \\ ${ }^{8}$ University of Wisconsin, 475 North Charter Street, Madison, WI 53706, USA \\ ${ }^{9}$ CRyA, Universidad Nacional Autónoma de México, Apdo. 3-72, 58090 Morelia, Michoacán, México \\ ${ }^{10}$ The Kavli Institute for Astronomy and Astrophysics, Peking University, Yi He Yuan Lu 5, Hai Dian Qu, Beijing 100871, China \\ ${ }^{11}$ University of Sheffield, Hicks Building, Hounsfixeld Road, Sheffield S3 7RH, UK \\ 12 Astronomical Institute, Utrecht University, Princetonplein 5, 3584CC Utrecht, The Netherlands \\ ${ }^{13}$ SUPA, Institute for Astronomy, University of Edinburgh, Royal Observatory Edinburgh, Blackford Hill, Edinburgh, EH9 3HJ, UK \\ Received 2009 October 18; accepted 2010 February 11; published 2010 March 29
}

\begin{abstract}
In this paper, we present VLT/SINFONI integral field spectroscopy of RCW 34 along with Spitzer/IRAC photometry of the surroundings. RCW 34 consists of three different regions. A large bubble has been detected in the IRAC images in which a cluster of intermediate- and low-mass class II objects is found. At the northern edge of this bubble, an $\mathrm{HII}$ region is located, ionized by $3 \mathrm{OB}$ stars, of which the most massive star has spectral type 08.5V. Intermediate-mass stars $\left(2-3 M_{\odot}\right)$ are detected of G- and K-spectral type. These stars are still in the premain-sequence (PMS) phase. North of the $\mathrm{H}$ II region, a photon-dominated region is present, marking the edge of a dense molecular cloud traced by $\mathrm{H}_{2}$ emission. Several class 0/I objects are associated with this cloud, indicating that star formation is still taking place. The distance to RCW 34 is revised to $2.5 \pm 0.2 \mathrm{kpc}$ and an age estimate of $2 \pm 1 \mathrm{Myr}$ is derived from the properties of the PMS stars inside the H II region. Between the class II sources in the bubble and the PMS stars in the H II region, no age difference could be detected with the present data. The presence of the class 0/I sources in the molecular cloud, however, suggests that the objects inside the molecular cloud are significantly younger. The most likely scenario for the formation of the three regions is that star formation propagated from south to north. First the bubble is formed, produced by intermediate- and low-mass stars only, after that, the $\mathrm{H}$ II region is formed from a dense core at the edge of the molecular cloud, resulting in the expansion similar to a champagne flow. More recently, star formation occurred in the rest of the molecular cloud. Two different formation scenarios are possible. (1) The bubble with the cluster of low- and intermediate-mass stars triggered the formation of the $\mathrm{O}$ star at the edge of the molecular cloud, which in its turn induces the current star formation in the molecular cloud. (2) An external triggering is responsible for the star formation propagating from south to north.
\end{abstract}

Key words: H II regions - infrared: stars - photon-dominated region (PDR) - stars: early-type - stars: formation stars: pre-main sequence

Online-only material: color figures

\section{INTRODUCTION}

One of the key questions in astrophysics is understanding the formation and early evolution of high-mass stars. They play a major role in shaping the interstellar medium due to their strong stellar winds and UV radiation fields. Observations show that high-mass stars preferentially form in clusters; therefore, if we wish to understand the formation of high-mass stars, we need to understand the clustered mode of star formation (Zinnecker \& Yorke 2007).

The actual formation of the massive stars happens deeply embedded in molecular clouds behind thousands of magnitudes of visual extinction and is only observable at far-infrared to radio wavelengths, providing indirect information about the

\footnotetext{
* Based on observations collected at the European Southern Observatory at Paranal, Chile (ESO program 078.C-0780).
}

stars themselves. As soon as massive stars begin to emit UV photons and start to develop a stellar wind, the surrounding molecular cloud evaporates and the stars become visible at shorter wavelengths.

For the direct detection and the study of the recently formed massive stars, the near-infrared is the most suitable regime. The emission of warm dust, the dominant source of emission at midinfrared wavelengths, is much fainter in the near-infrared and the actual photospheres of the high-mass stars can be probed. The success of this approach is demonstrated by several near-infrared imaging studies (e.g., Hanson et al. 1997; Blum et al. 2000; Feldt et al. 2003; Alvarez et al. 2004; Bik 2004, L. Kaper et al. 2010, in preparation). These imaging studies reveal the stellar content hidden in those star-forming regions, but also show the limitations, as these studies are limited to regions with $A_{V}$ less than $\sim 30$ mag. Near-infrared spectroscopy, however, results in 
a much less ambiguous identification and classification of the massive star content (e.g., Watson \& Hanson 1997; Blum et al. 2000; Hanson et al. 2002; Kendall et al. 2003; Bik et al. 2005; Puga et al. 2006). These studies demonstrate the diagnostic power of near-infrared spectroscopy in characterizing the young, massive, stellar population.

Previous near-IR spectroscopic studies only considered a limited number of stars per cluster. This incomplete spectroscopic sampling of the stellar population, as well as the complexity of the fields, prevents any systematic investigation of the stellar content in the context of their environment. A systematic study on how the stellar content depends on the cluster properties like total luminosity, evolutionary phase as well as their environment requires a full spectroscopic census of the high-mass stellar content.

We started a program to obtain a full near-infrared spectroscopic census of the stellar content of a sample of high-mass star-forming regions by means of integral field spectroscopy using SINFONI at the Very Large Telescope (VLT). Integral field spectroscopy provides a spectrum of every spatial element in the field of view. This yields not only a spectrum of all the stars in the observed area, but also emission line images of $\mathrm{H}$ II regions, photon-dominated regions (PDRs), and outflows. Supporting Spitzer IRAC and MIPS imaging reveal the more deeply embedded objects and enable the classification of young stellar objects via their colors.

This paper is the first in a series of studies presenting our integral field data set of 10 high-mass star-forming regions. As a subject of this first study, we chose the region RCW 34, associated with the Vela Molecular Ridge (VMR). The region consists of a well-developed $\mathrm{H}$ II region interacting with a dense molecular cloud to the north.

The interaction between the $\mathrm{H}$ II region and the molecular cloud suggests that a triggered star formation scenario could be applicable to RCW 34. Three different mechanisms are discussed in the literature, all resulting from the presence of an expanding $\mathrm{H}$ II region inside a molecular cloud (Elmegreen 1998; Deharveng et al. 2005). The first mechanism relies on the presence of pre-existing molecular clumps. The high pressure of the HiI region can result in the collapse of those clumps and the creation of cometary globules forming a bright rim. The second mechanism also produces such globules and bright rims; however, the clumps are not pre-existing but created by sweeping up gas by the $\mathrm{H}$ in region. The cores become unstable on short timescales and are slowly eroded away by the $\mathrm{H}$ II region. The third mechanism is the "collect and collapse" mechanism (Elmegreen \& Lada 1977). Similar to the second mechanism, the HiI region sweeps up neutral gas; however, in this scenario the neutral layer becomes unstable on longer timescales and very massive cores are formed. When these massive cores collapse they form new star clusters.

We will determine the properties (spectral type, mass, and age) of the high- and intermediate-mass stellar population of RCW 34 by means of near-infrared integral field spectroscopy and Spitzer/IRAC images. The cluster environment is characterized via $\mathrm{H}$ II, $\mathrm{He}$ I, and $\mathrm{H}_{2}$ emission lines. The three triggering mechanisms are tested on the observed properties of RCW 34, and it turns out that an unusual mode of triggering might be at work here.

\section{1. $R C W 34$}

The HII region RCW 34 (Rodgers et al. 1960; IRAS 08546-4254 or Gum 29, Gum 1955) is located in the VMR cloud C (Liseau et al. 1992). The region is an optically visible $\mathrm{H}$ II region of about $2^{\prime} \times 2^{\prime}$ in extent and slightly offset from the main dense cores defining the VMR cloud C detected by CO observations (Murphy \& May 1991; Yamaguchi et al. 1999). Due to a similar velocity $\left(v_{\mathrm{lsr}}=6 \mathrm{~km} \mathrm{~s}^{-1}\right)$, Murphy \& May (1991) argue that RCW 34 is likely part of the C-cloud. A similar velocity has been measured from the CS line $\left(v_{\mathrm{lsr}}=5.5 \mathrm{~km} \mathrm{~s}^{-1}\right.$; Bronfman et al. 1996), confirming the association with cloud C, which would place the region at a distance somewhere between 1 and $2 \mathrm{kpc}$.

Herbst (1975) and van den Bergh \& Herbst (1975) allowed that the exciting star of RCW 34 might be more distant, but favored an association with the Vela-R2 R-association at a distance of $870 \mathrm{pc}$. Optical spectroscopy by Heydari-Malayeri (1988) implies that the central source vdBH 25a (van den Bergh \& Herbst 1975) is of spectral type O8.5V. This suggests a distance of $2.9 \mathrm{kpc}$, supporting the idea that RCW 34 is more distant than Vela-R2. In Section 4.1, we use near-infrared spectroscopy of the cluster OB stars to derive a distance in rough agreement with Heydari-Malayeri (1988). Heydari-Malayeri also detected a north-south velocity gradient in the ionized gas, which he suggested to be evidence that RCW 34 is an $\mathrm{H}$ II region undergoing a champagne flow. This is supported by Deharveng et al. (2005) who conclude, based on the Midcourse Space Experiment and near-infrared observations, that the region is not the result of the collect and collapse triggered star formation scenario.

Several signs of active star formation are present in RCW 34. Two water masers have been reported (Braz \& Epchtein 1983; Caswell et al. 1989) as well as a class II methanol maser (Chan et al. 1996; Walsh et al. 1997). Associated with the water masers, CS emission has been found (Zinchenko et al. 1995) with an estimated mass of $1320 M_{\odot}$. Gyulbudaghian \& May (2007) reported the detection of $1.2 \mathrm{~mm}$ continuum emission toward RCW 34.

The outline of the paper is as follows. Section 2 outlines the data reduction of the SINFONI and Spitzer/IRAC data sets used in this paper. In Section 3, the interaction between the H II region and the cluster surroundings is studied using the IRAC data as well as the SINFONI emission line maps. Section 4 focuses on the stellar spectra obtained in the SINFONI field; spectral types, luminosity class as well as extinctions are derived; and in Section 5, the stellar content is discussed and an age estimate is obtained for the NIR cluster in RCW 34. In Section 6, we discuss the complete region and combine all the different properties to derive a possible formation scenario for RCW 34; and Section 7 summarizes the main conclusions of the paper.

\section{OBSERVATIONS AND DATA REDUCTION}

\subsection{SINFONI Observations}

Near-infrared $H$ - and $K$-band observations were performed using the Integral Field instrument SINFONI (Eisenhauer et al. 2003; Bonnet et al. 2004) on UT4 (Yepun) of the VLT at Paranal, Chile. The observations of RCW 34 were performed in service mode between 2007 February 4 and 9. The non-AO mode of SINFONI, used in combination with the setting providing the widest field of view $\left(8^{\prime \prime} \times 8^{\prime \prime}\right)$, delivers a spatial resolution of 0 '.25 per slitlet. The typical seeing during the observations was 0.7 in $K$. The $\mathrm{H}+\mathrm{K}$ grating was used, covering these bands with a resolution of $R=1500$ in a single exposure.

We observed RCW 34 with a detector integration time (DIT) of $30 \mathrm{~s}$ per pointing to guarantee a good signal-to-noise ratio 
$(\mathrm{S} / \mathrm{N} \sim 70)$ for the early B-type stars within this cluster. RCW 34 is much larger than the field of view of SINFONI, the near-infrared extent of the H II region is $104^{\prime \prime} \times 60^{\prime \prime}$ on the sky. The observations were centered on the central $08.5 \mathrm{~V}$ star $\left(\right.$ vdBH 25a) at coordinates: $\alpha(2000)=08^{\mathrm{h}} 56^{\mathrm{m}} 28^{\mathrm{s}} .1, \delta(2000)=$ $-43^{\circ} 05^{\prime} 55^{\prime \prime} 6$. We observed this area with SINFONI using a raster pattern, covering every location in the cluster at least twice. The offset in the east-west direction was $4^{\prime \prime}$ (i.e., half the field of view of the detector) and the offset in the north-south direction was 6".75. A sky frame was taken every 3 minutes using the same DIT as the science observations. The sky positions were chosen based on existing NIR imaging in order to avoid contamination. Immediately after every science observation, a telluric standard of B spectral type was observed, matching as close as possible the air mass of the object. This star is used for the removal of the telluric absorption lines as well as for flux calibration. The stars chosen as telluric standards are HIP 048652, HIP 045117, HIP 052202, and HIP 040629, one for each of the observing blocks.

\subsubsection{Data Reduction}

The SINFONI data were reduced using the SPRED (version 1.37) software developed by the MPE SINFONI consortium (Schreiber et al. 2004; Abuter et al. 2006). This software was used to apply all the basic steps of the data reduction like flat-field correction, dark removal, distortion correction, wavelength calibration, and correction of bad pixels. Special care was taken to remove bad pixels from the calibration frames. The construction of the final calibration data was done in three iterations by first constructing a preliminary calibration set used to detect and remove the bad pixels using the three-dimensional (3D) information. The cleaned raw files are used to construct a new calibration set and the procedure is repeated.

The bad pixels on the science data are removed using the same 3D interpolation method. However, due to severe cross talk between the pixels, the surrounding pixels are affected at lower intensity and are not properly removed by the algorithm. Therefore, we apply an additional bad pixel removal later in the reduction process. The cleaned science and sky frames are reconstructed into 3D cubes using the calibration data. Also the dark frames are subtracted and the cubes are corrected for atmospheric diffraction. We applied the procedure described by Davies (2007) to remove $\mathrm{OH}$ line residuals.

In the reduced cubes, we discovered a drift of the stellar position versus wavelength. The shift is strongest in $K$ (up to 2 pixel) and resulted in an elongation of the stars in the collapsed image. We measured the position of the telluric standard star versus wavelength using a two-dimensional Gaussian and fitted a spline to the change of position in both spatial directions. This spline fit was used to correct the science cubes. The residual measured on some bright stars in the science frames was within 0.5 pixel, which is acceptable for our purposes.

The last step in the reduction process is flux calibration and removal of the telluric absorption lines. This is done using the extracted spectrum of a B-type standard star, using an aperture radius of 6 pixel $(0.75)$. After the extraction, the correction for the telluric absorption lines in the science spectra is done in two steps: first, the telluric standard star is corrected using a high signal-to-noise atmospheric spectrum. ${ }^{14}$ This high $\mathrm{S} / \mathrm{N}$ spectrum is not taken at the same air mass and

\footnotetext{
14 Taken from the SINFONI Web site and obtained at NSO/Kitt Peak Observatory.
}

atmospheric conditions as our science spectra, but provides a first rudimentary correction in order to reduce the absorption contamination of the telluric standard star.

Second, the Brackett and $\mathrm{He} \mathrm{I}$ lines are fitted with a Lorentzian profile. The line-fits are subtracted from the original telluric standard star spectrum, leaving an atmosphere transmission spectrum. The flux calibration of the spectra uses the Two Micron All Sky Survey (2MASS; Cohen et al. 2003) magnitude of the standard stars. As we only use normalized spectra for the point sources and flux ratios for the emission line spectra, the uncertainty in the flux calibration due to atmospheric variations is not critical.

The correction of the atmospheric absorption lines and the flux calibration are performed on the 3D cubes; the spectrum of every spatial pixel is divided by the spectrum of the telluric standard star and flux calibrated. Finally, the individual 3D cubes of one observing block (between 50 and 60 cubes) were combined into one large 3D cube. For RCW 34, four of those large cubes are needed to cover the near-IR emission of the cluster.

Before extracting the point source spectra from the science cubes, an attempt was made to remove the extended background emission from the data. This background emission is primarily nebular emission from the H II region and the PDR (Brackett lines, $\mathrm{He} \mathrm{I}$, and $\mathrm{H}_{2}$ ). A median filter with a kernel of 5 pixel is used to remove the extended nebular component in the cubes. We applied this median filter 3 times to remove any high frequency variations present which could be due to stars. This median filtering has only a small effect on the stellar continuum; the contribution in the total flux is less than $1 \%$. The point sources were extracted using a circular aperture with a radius of 6 pixel, analogous to that of the standard star.

Several maps centered on emission lines as well as continuum maps are created from the combined SINFONI data cubes. The maps are made by summing 4-7 slices of the data cube in the wavelength direction. The images in the spectral lines are corrected for continuum emission from the stars by interpolating each pixel of the adjacent continuum images (blueward and redward of the line, respectively). This results in a clean subtraction for most of the stars. However, for the brightest stars in the maps, residuals remain present. An interpolation of the line emission a bit further away from the stars successfully restored these residuals.

We created velocity maps of the strongest emission lines, $\mathrm{Br} \gamma$ and $\mathrm{H}_{2}$, for every (spatial) pixel in the data cube. The central velocity of the $\mathrm{Br} \gamma$ and $\mathrm{H}_{2}$ emission line is derived from a Gaussian fit to the line profile. To obtain better accuracy in the wavelength calibration, we performed the same fitting procedure to a nearby $\mathrm{OH}$ skyline. Finally, the velocities were converted to the local standard of rest. The errors were calculated by quadratically summing the fitting errors on the nebular line and the $\mathrm{OH}$ line and are on the order of $10-15 \mathrm{~km} \mathrm{~s}^{-1}$.

\subsection{IRAC Data Reduction and Photometry}

Additional data obtained with IRAC (Fazio et al. 2004) on board of the Spitzer satellite have been retrieved from the Spitzer archive (PID: 40791, PI: Majewski). The data were obtained on 2008 January 31 in a mapping mode, covering a large part of the outer galaxy. We selected the frames covering the area around the cluster as well as an adjacent control field. Each frame has an exposure time of $1.2 \mathrm{~s}$ and every position is covered twice. The raw frames were processed using the standard pipeline version S17.02 to produce the basic calibration data set. This data set 


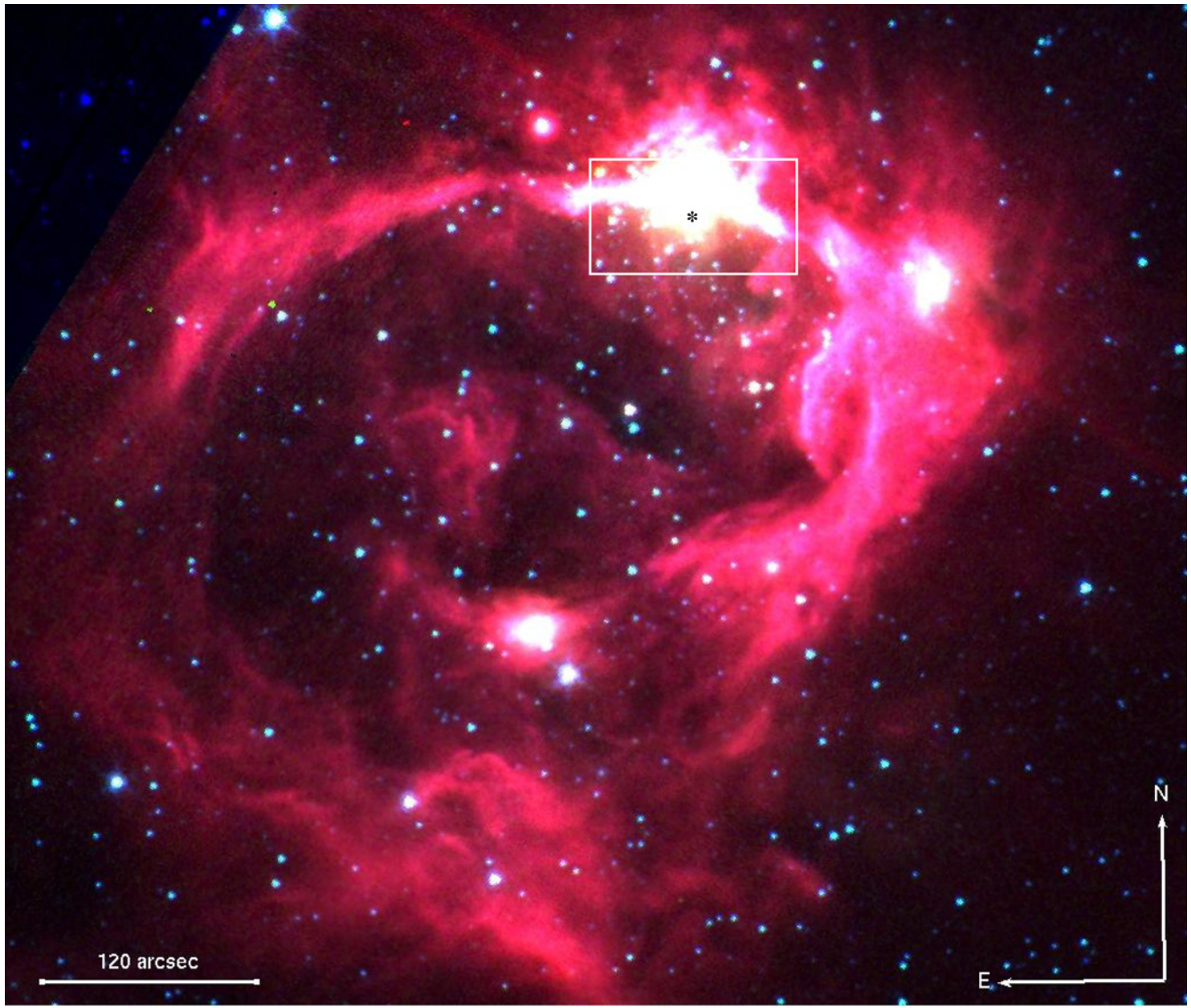

Figure 1. Spitzer/IRAC three-color image of RCW 34 and its surroundings (blue: $3.6 \mu \mathrm{m}$, green: $4.5 \mu \mathrm{m}$, red: $8 \mu \mathrm{m}$, color version online). The top left corner is only covered at $3.6 \mu \mathrm{m}$. The area delimited by the white box is the area covered by the SINFONI observations. The location of the central star of RCW 34 , vdBH $25 \mathrm{a}$ $\left(\alpha(2000)=08^{\mathrm{h}} 56^{\mathrm{m}} 28^{\mathrm{s}} .1, \delta(2000)=-43^{\circ} 05^{\prime} 55^{\prime \prime}\right.$. 6 .), is shown with an asterisk. The $\mathrm{H}$ II region surrounds vdBH $25 \mathrm{a}$ and is fully covered by our SINFONI observations. (A color version of this figure is available in the online journal.)

was processed further using MOPEX version 16.2.1 (Makovoz et al. 2006).

As the IRAC data are undersampled, aperture photometry is preferred above psf-fitting photometry. Source detection and photometry were performed using the Daophot package under IRAF. Point sources with a peak level of more than 5 times the rms of the background were detected. Aperture photometry was applied with an aperture of 3 pixel (3.7) and a sky annulus between $3\left(3^{\prime \prime} 7\right)$ and $7\left(8^{\prime \prime} .5\right)$ pixel. These small values were chosen due to the variable extended emission. The appropriate aperture corrections and zero points were adopted (Tables 5.1 and 5.7 of the IRAC Data Handbook) to obtain the magnitudes of the objects.

\section{CLUSTER SURROUNDINGS}

NIR imaging (Bik 2004; Deharveng et al. 2005) shows an H II region extending about $1^{\prime} .5 \times 1^{\prime}$ around the central star $\mathrm{vdBH}$ 25a. The IRAC images (Figure 1) show a much larger starforming region than is visible in the NIR. The region extends to the south and east and shows an open bubble structure. The total size of the mid-IR region is about $6^{\prime} \times 9^{\prime}$. This structure looks similar to the (partial) bubbles identified in the GLIMPSE survey (Churchwell et al. 2006).

In this section, we will determine the nature of the stars located inside the bubble using the IRAC photometry and investigate whether the objects in the bubble are related to those detected in the $\mathrm{H}$ II region. We analyze the extended emission seen in the SINFONI observations and derive properties of the $\mathrm{H}$ II region and the surrounding molecular cloud.

\subsection{Spitzer/IRAC Imaging of the Cluster and its Surroundings}

A three-color Spitzer/IRAC image of the region is shown in Figure 1 and, Figure 2 shows a [3.6]-[4.5] versus [5.8]-[8.0] color-color diagram of the stars in the field. Objects not detected in all bands are not included in the analysis. We have not plotted the stars in the control field which only contains stars without IR excess. With the color criteria proposed by Megeath et al. (2004) and Allen et al. (2004), we can identify several classes of objects. Stars without IR excess have colors close to 0.0. The IRAC colors are insensitive to the effective temperature of the photospheres. These objects, plotted as starshaped symbols, could be fore- or background late-type stars as well as earlier-type main-sequence stars associated with the star-forming region. The objects with [3.6]-[4.5] between 0.0 and 0.8 and [5.8]-[8.0] between 0.4 and 1.1 are classified as class II objects. This region is marked with a box, and the objects showing class II colors are plotted as triangles. Objects with [3.6]-[4.5]>0.8 and [5.8]-[8.0] $>0.0$ or [3.6]-[4.5] $>$ 0.4 and [5.8]-[8.0] $>1.1$ are classified as protostars (class 0 and I objects; diamonds), objects with [5.8]-[8.0] $>1.1$ and 


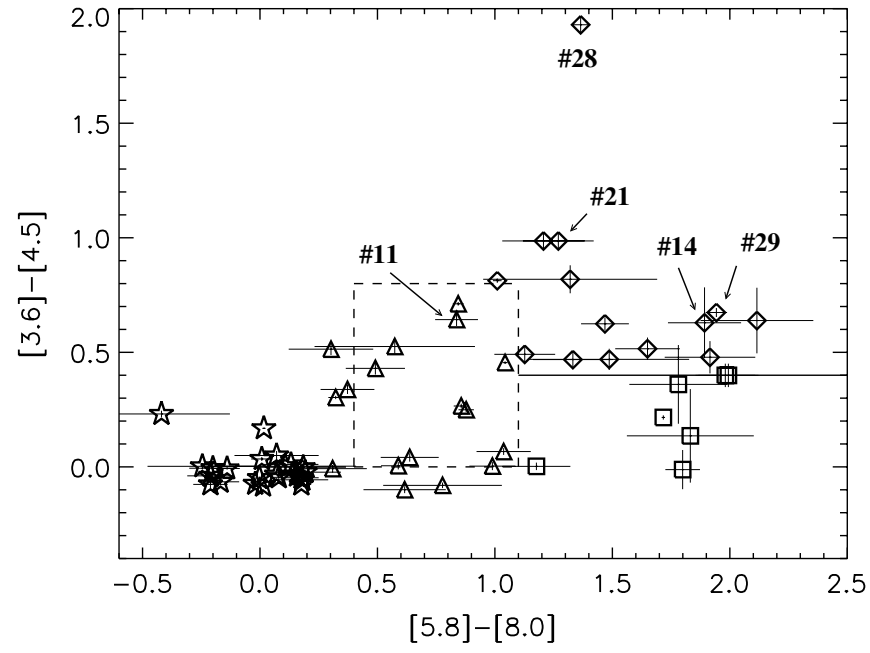

Figure 2. Spitzer/IRAC color-color diagram. The star-shaped points represent stars without infrared excess, class II stars are plotted as triangles inside the squared box (Megeath et al. 2004). The diamond symbols represent the location of the protostars (class $0 / \mathrm{I}$ ) and the squares show the location of the class I/II sources. The numbered objects are located inside the SINFONI field and are discussed in more detail in Section 4.3.

[3.6]-[4.5] between 0.0 and 0.4 are classified as class I/II sources (square symbols). The five numbered objects are located in the SINFONI field and are discussed in more detail in Sections 4.3. and 5.3.

The class I/II identification should be considered with caution. Megeath et al. (2004) show that these objects are likely class II sources with the $8 \mu \mathrm{m}$ flux contaminated by variable extended emission. Figure 1 shows that these objects are located on top of a strong diffuse background. Therefore, we consider them as class II objects. Additional sources of contamination, especially for the detection of protostars, could be planetary nebula, AGB stars, or background galaxies (Whitney et al. 2003). However, the absence of sources with these colors in the control field suggests that the contamination is negligible. We have plotted the different classes on the IRAC $3.6 \mu \mathrm{m}$ image (Figure 3) to study their potential relation with the extended MIR emission of the bubble. The locations of the stars without infrared excess (white, star shapes) are not related to the extended emission, but evenly distributed over the field outside the bubble. These objects are most likely field stars.

The location of the young stellar objects shows a clear correlation with the bubble and the extended emission. Most of the class II sources (yellow triangles) are located inside the bubble. The protostars (red diamonds) are spatially associated with the extended MIR emission. Most of these objects are located near the bright arc in the north, but also some objects are associated with the fainter extended emission in the southern part of the bubble. Although no obvious cluster is visible inside the bubble, the class II objects located inside the bubble suggest a recent star formation event. This might have been responsible for the creation of the bubble (Churchwell et al. 2006). The location of the younger protostars in the outer regions of the bubble indicates the presence of a more recent generation of stars near the edge of the bubble.

\subsection{SINFONI Line and Continuum Maps}

The SINFONI observations are centered on the ionizing source, vdBH $25 \mathrm{a}$, and cover both the $\mathrm{H}$ II region as well as the bright arc seen in the IRAC images. Figure 4 shows the

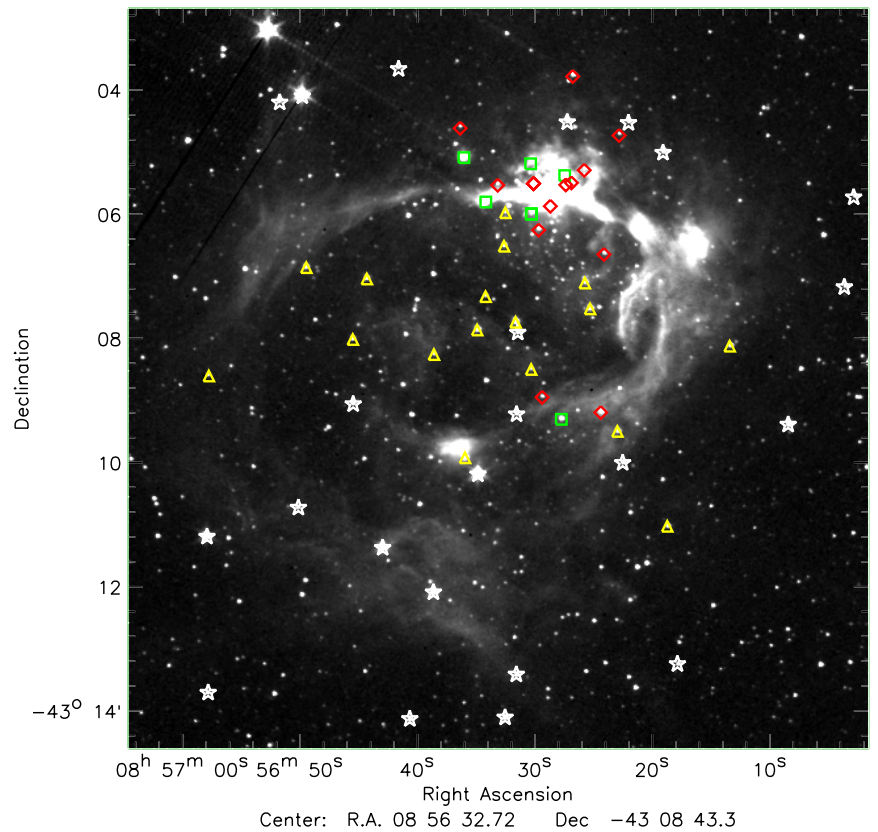

Figure 3. Spitzer/IRAC $3.6 \mu \mathrm{m}$ image of RCW 34 and its surroundings. The different classes of objects as derived from the IRAC color-color diagram are overplotted. The symbols used are identical to those in Figure 2.

(A color version of this figure is available in the online journal.)

three-color composite of three line maps along with contours of the IRAC $3.6 \mu \mathrm{m}$ image. This image reveals the stellar content of this region and the overlay shows that several near-infrared sources are also detected at IRAC wavelengths. The region is dominated by the two bright stars in the center of the field (one of them is vdBH 25a at the location of the asterisk symbol) and a relatively sparse cluster of about 130 stars down to $K=17 \mathrm{mag}$, surrounding the central region. For all the point sources visible in this image, a SINFONI $H$ - and $K$-band spectrum is available and a spectral classification is obtained (Section 4). The H II region is traced by $\mathrm{Br} \gamma(2.16 \mu \mathrm{m}$, red) and $\mathrm{He} \mathrm{I}(2.058 \mu \mathrm{m}$, blue) emission and the surrounding PDR by the molecular $\mathrm{H}_{2}$ emission $(2.12 \mu \mathrm{m}$, green). Just north of the position of the central star, the $\mathrm{Br} \gamma$ emission is strongest. Even further to the north, the $\mathrm{Br} \gamma$ and $\mathrm{He} \mathrm{I}$ emission drop abruptly and the $\mathrm{H}_{2}$ emission begins, indicating the interface between the ionized and the molecular gas, coincident with the bright arc seen in the IRAC images.

\subsubsection{H II Region}

We have extracted spectra at six locations in the $\mathrm{H}$ II region covering most of the $\mathrm{H}$ II region (annotated with numbers I-VI in Figure 4). Table 1 gives the positions and sizes of the six extracted regions.

As an example, the upper panels of Figure 5 show the spectra extracted from region II, just north of the ionizing star.

To check the hypothesis of a champagne flow, we estimate the column density of the molecular cloud in which the H II region is embedded. In an $\mathrm{H}$ II region, the relative line intensities of the hydrogen recombination lines are mostly determined by the Einstein coefficients, with a very weak dependence on density and electron temperature (Storey \& Hummer 1995). For example, the ratio of the $\operatorname{Br} 10(1.73 \mu \mathrm{m})$ and the $\mathrm{Br} \gamma$ $(2.16 \mu \mathrm{m})$ lines is predicted to be 0.33 . Deviations from this value are caused by foreground extinction. We have converted the measured $\mathrm{Br} 10 / \mathrm{Br} \gamma$ ratio to $A_{V}$ using the Cardelli et al. 


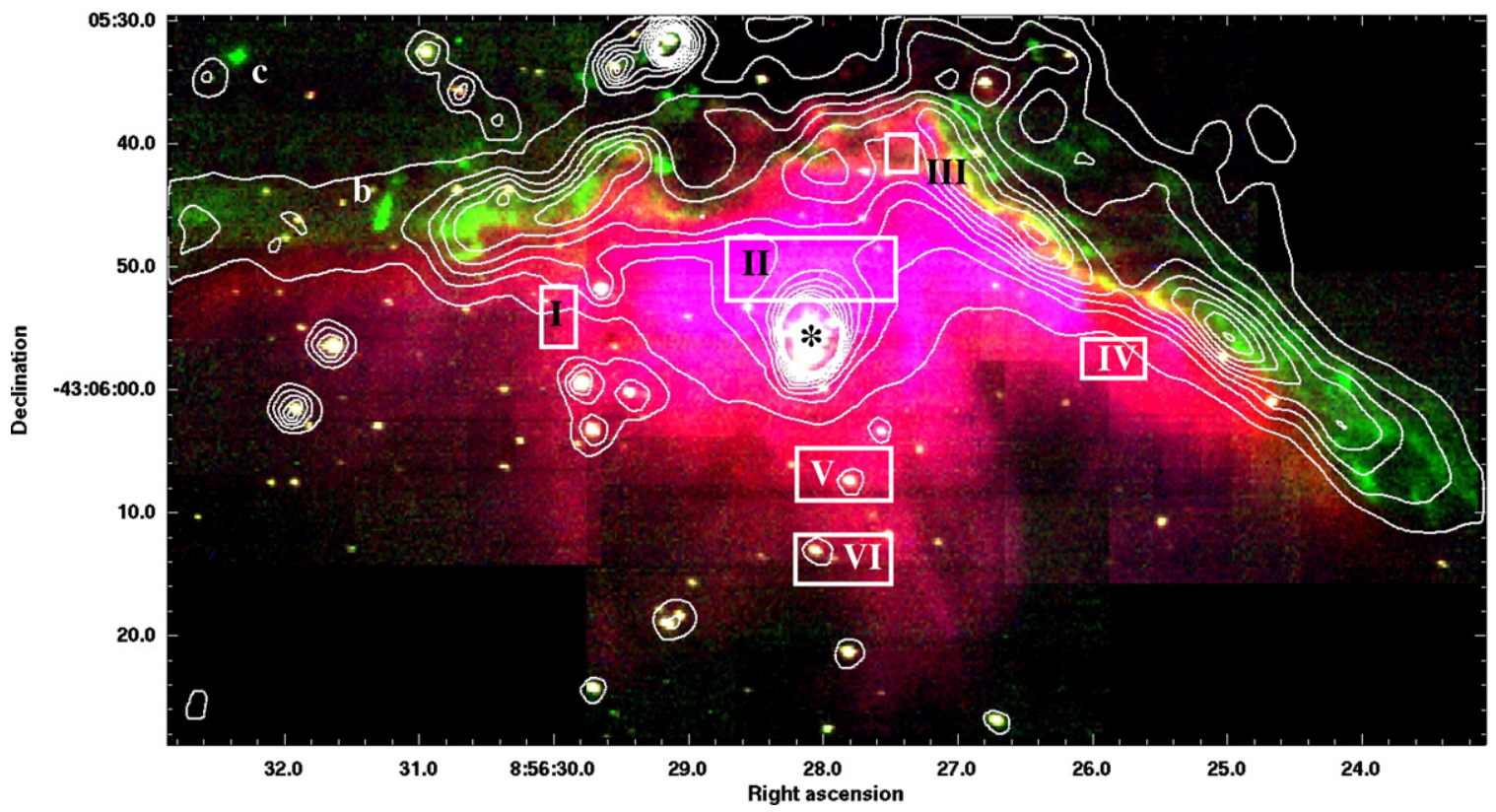

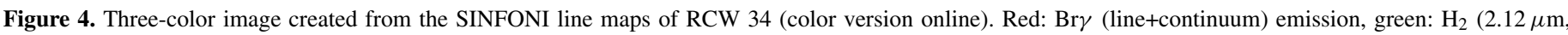

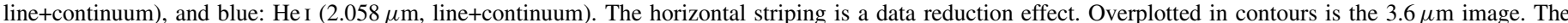

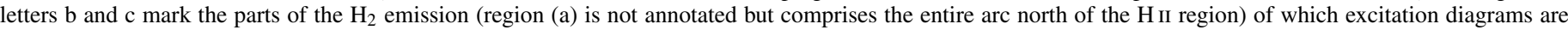

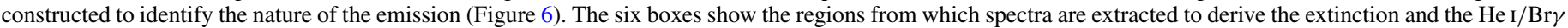
ratio. The asterisk shows the location of the main ionizing source vdBH $25 \mathrm{a}$.

(A color version of this figure is available in the online journal.)

Table 1

Properties of the H II Gas

\begin{tabular}{|c|c|c|c|c|c|c|}
\hline Region & $\begin{array}{l}\text { R.A. (2000) } \\
\quad(\text { h m s) }\end{array}$ & $\begin{array}{c}\text { Decl. (2000) } \\
\left({ }^{\circ} \prime \prime \prime\right)\end{array}$ & $\begin{array}{c}\text { Size } \\
\left({ }^{\prime \prime}\right)\end{array}$ & $\begin{array}{c}v_{\mathrm{lsr}} \\
\left(\mathrm{km} \mathrm{s}^{-1}\right)\end{array}$ & $\mathrm{He} \mathrm{I} / \mathrm{Br} \gamma$ & $\begin{array}{c}A_{V} \\
(\mathrm{mag})\end{array}$ \\
\hline I & 085629.8 & -430552.4 & $2.4 \times 4.6$ & $18 \pm 13$ & $<0.13$ & $5.3 \pm 0.2$ \\
\hline II & 085628.1 & -430550.8 & $13.7 \times 4.9$ & $16 \pm 8$ & $0.038 \pm 0.002$ & $5.1 \pm 0.1$ \\
\hline III & 085627.3 & -430539.2 & $2.3 \times 2.7$ & $16 \pm 9$ & $<0.17$ & $15.9 \pm 0.6$ \\
\hline IV & 085625.7 & -430555.7 & $5.0 \times 3.0$ & $10 \pm 12$ & $0.032 \pm 0.006$ & $5.6 \pm 0.2$ \\
\hline V & 085627.7 & -430605.0 & $7.7 \times 4.0$ & $13 \pm 9$ & $0.047 \pm 0.004$ & $4.4 \pm 0.1$ \\
\hline VI & 085627.7 & -430611.9 & $7.7 \times 3.7$ & $11 \pm 10$ & $0.06 \pm 0.01$ & $1.4 \pm 0.2$ \\
\hline
\end{tabular}

(1989) extinction law. The derived $A_{V}$ values for the six extracted regions are listed in the Column 7 of Table 1 . The bulk of the $\mathrm{H}$ II region has an $A_{V}$ of around 5-6 mag (regions I, II, and IV). In region III, on the border with the molecular cloud, we measure an $A_{V}$ of $15.9 \mathrm{mag}$, while toward the south the extinction declines to $4.4 \mathrm{mag}$ and $1.5 \mathrm{mag}$ for regions $\mathrm{V}$ and VI, respectively. This extinction gradient can be explained as a density gradient in the surrounding molecular cloud and is consistent with the presence of a champagne flow.

The (extinction corrected) ratio of the $1.70 \mu \mathrm{m}$ He I line over $\mathrm{Br} \gamma$ is dependent on the temperature of the ionizing star and can be used to constrain its spectral type (Lumsden et al. 2003). The extinction correction line ratios for the six regions are given in the Column 6 of Table 1. The maximum ratio is measured just south of the location of the ionizing stars, and has a value of $0.047 \pm 0.004$ in region $\mathrm{V}$ and $0.06 \pm 0.01$ in region VI. Comparing this value to the predictions of Lumsden et al. (2003), an effective temperature for the central star around $35,000 \mathrm{~K}$ is expected, which corresponds to a spectral type between $\mathrm{O} 8 \mathrm{~V}$ and $\mathrm{O} 9 \mathrm{~V}$ (Martins et al. 2002), consistent with the optical spectral type determination of Heydari-Malayeri (1988).

\subsubsection{PDR and Outflows}

The IRAC detections of class 0 and I sources shows that stars are still forming close to the bright arc. An additional argument for active star formation is the presence of outflows, detectable by $\mathrm{H}_{2}$ line emission (bottom panels of Figure 5)

The $\mathrm{H}_{2}$ emission in the near-IR can be either thermally or non-thermally excited. The thermal emission originates from shocked neutral gas being heated up to a few $1000 \mathrm{~K}$ (outflows), while the non-thermal emission is due to fluorescence by non-ionizing UV photons between 912 and $1108 \AA$. These two mechanisms can be distinguished as they preferentially populate different energy levels giving rise to different line ratios. The fluorescence mechanism preferably excites the higher vibrational levels, while for thermal excitation the lower levels are preferred (Davis et al. 2003).

Figure 4 shows the location of the $2.12 \mu \mathrm{m} \mathrm{H}_{2}$ emission (green) in relation to the emission from ionized gas $(\mathrm{Br} \gamma$ and $\mathrm{He} \mathrm{I}$ ). The $\mathrm{H}_{2}$ emission has a very filamentary structure and appears just north of the ionized gas, co-located with the bright arc in the IRAC images. Additionally, two compact sources are visible in the northeast. To identify the excitation mechanism of the arc of filamentary emission (region (a)) as well as that of the 

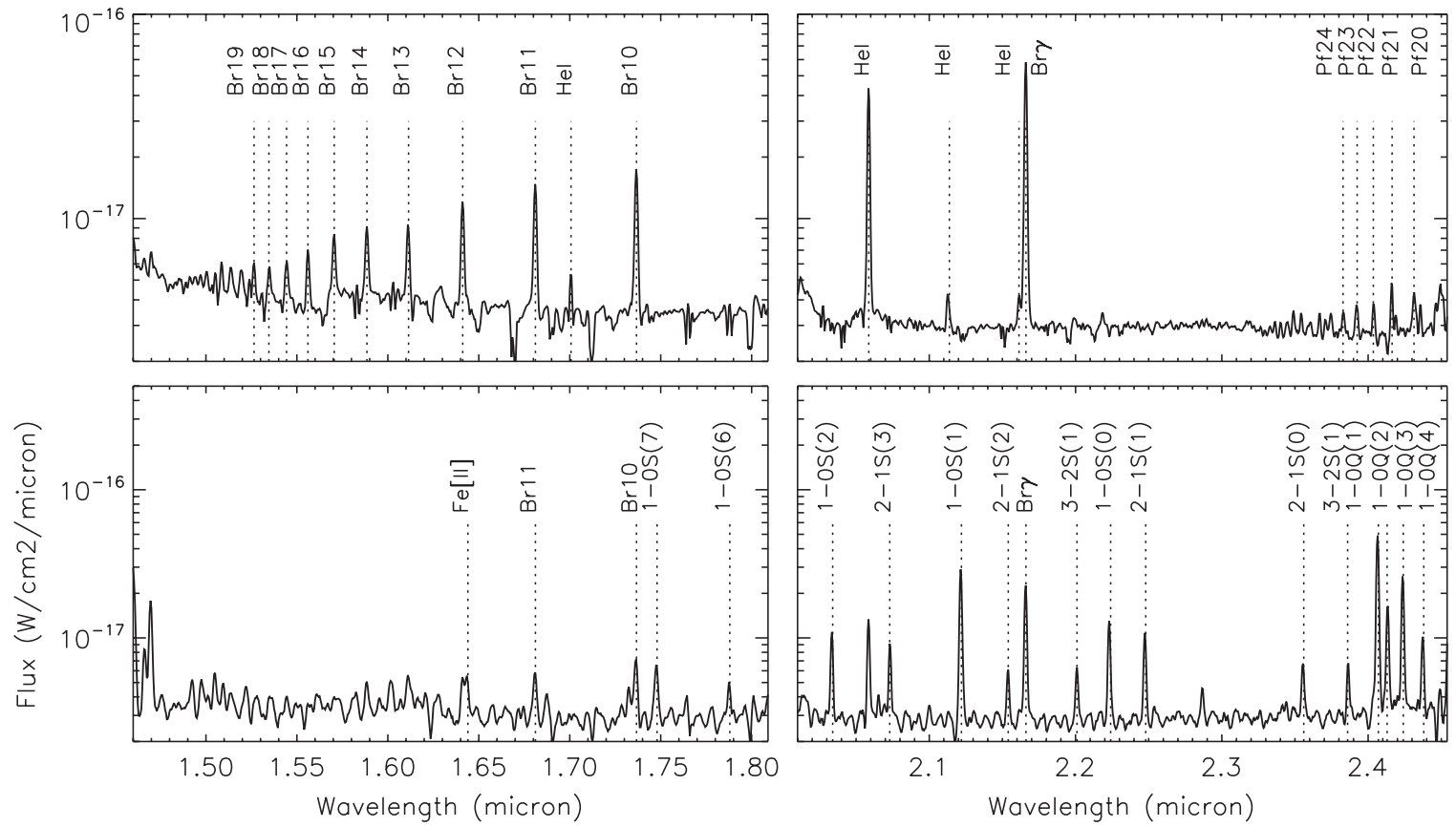

Figure 5. Top panels: $H$ - and $K$-band SINFONI spectra of the H II region in RCW 34 . The spectra are drawn from a region just north of the ionizing stars (region II in Figure 4). The spectra show numerous hydrogen lines of which $\operatorname{Br} \gamma(2.166 \mu \mathrm{m})$ is the most prominent. Also several He I lines are visible, suggesting that the central star is of spectral type late O. Bottom panels: spectra of the PDR, north of the $\mathrm{H}$ II region (region (a)). The spectrum is dominated by $\mathrm{H}_{2}$ lines. Also some faint [Fe II] emission is present. This line can be formed in J-shocks and also in the low density material between the H II region and the PDR.

Table 2

Physical Properties of the $\mathrm{H}_{2}$ Gas

\begin{tabular}{llccr}
\hline \hline Region & $\begin{array}{c}T_{\text {rot }} \\
(\mathrm{K})\end{array}$ & $\begin{array}{c}N\left(\mathrm{H}_{2}\right) \\
\left(\mathrm{cm}^{-2}\right)\end{array}$ & $\begin{array}{c}A_{V} \\
(\mathrm{mag})\end{array}$ & \multicolumn{1}{c}{$\begin{array}{c}v_{\text {lsr }} \\
\left(\mathrm{km} \mathrm{s}^{-1}\right)\end{array}$} \\
\hline $\mathrm{a}$ & $3720 \pm 90$ & $3.6 \pm 0.6 \times 10^{17}$ & 9 & $+1 \pm 14$ \\
$\mathrm{~b}$ & $2217 \pm 90$ & $1.8 \pm 0.4 \times 10^{18}$ & 13 & $-14 \pm 14$ \\
$\mathrm{c}$ & $1980 \pm 115$ & $4.6 \pm 2.0 \times 10^{18}$ & 20 & $-26 \pm 16$ \\
\hline
\end{tabular}

two compact regions ((b) and (c), top left panel of Figure 6), we construct excitation diagrams for these regions.

First, the line flux ratios are extinction corrected. The ratio of the $1-0 \mathrm{~S}(1)(2.12 \mu \mathrm{m})$ over the $1-0 \mathrm{Q}(3)(2.423 \mu \mathrm{m})$ is insensitive to the ionization mechanism and has an expected value of 0.7 (Turner et al. 1977). Therefore, the observed ratios are used for an extinction estimate in the same manner as discussed in Section 3.2.1 (Table 2). Region (a) covers a large area, $262 \operatorname{arcsec}^{2}$ and shows a large-extinction variation. The derived properties are dominated by the strongest emission regions, located close to the $\mathrm{H}$ II region where the extinction is the lowest.

Figure 6 also show the excitation diagrams of the three regions. In these diagrams, the measured column densities of the lines are plotted against the energy of the upper level (see Martin-Hernández et al. 2008, for a detailed description of this diagram). The total column density was calculated using the description of Zhang et al. (1998). Different vibrational levels are indicated with different symbols. Region (a) has the most lines detected as it covers the largest area (the complete arc). For the spectra of regions (b) and (c), the weaker lines seen in the spectrum of region (a) are not detected and $3 \sigma$ upper limits are given instead. The solid line in the diagrams is a single temperature fit to all the data points, while the dashed line in the diagram of region (a) represents a linear fit to only the $1-0 \mathrm{~S}$ lines. For region (a), it is clear that the column densities are not represented by a single temperature gas, but that the line fluxes of the 2-1 and 3-2 vibrational levels are higher than expected from the 1-0 line fluxes. A likely excitation mechanism of the $\mathrm{H}_{2}$ gas in the arc is fluorescence by nonionizing UV photons. We have searched for spatial variations of the temperature and column density in region (a) by calculating the excitation diagrams for several sub-regions, but could not detect any significant deviations from the values of the total area.

The excitation diagrams of regions (b) and (c) are well represented by a single temperature. We note, however, that the 3-2 lines are not detected in either region, and the 2-1 lines are mostly upper limits. We conclude that the excitation diagrams of both regions are consistent with shock excited emission in an outflow. Their elongated shapes, especially for region (b), are also suggestive of outflow. The linear fits of the excitation diagrams also allow us to determine the column density and temperature of the emitting gas (Table 2). An additional constraint on the nature of the $\mathrm{H}_{2}$ emission regions is the velocity of the different regions.

The obtained velocities are listed in the last column of Table 2. The two outflows have a clear blueshifted velocity compared to that of the extended PDR, with region (c) having the largest value of $-26 \pm 16 \mathrm{~km} \mathrm{~s}^{-1}$. These relatively low velocities as well as the absence of [Fe II] at $1.644 \mu \mathrm{m}$ suggest that both shocks are C-type shocks (Stahler \& Palla 2005). The more destructive shocks (J-type) would have strong [Fe II] emission as well as larger shock velocities (Hollenbach \& McKee 1989). The velocity measured in the arc (region (a)) of $1 \pm 14 \mathrm{~km} \mathrm{~s}^{-1}$ is more blueshifted than the velocity of the HII region, but consistent with that of the Vela molecular cloud: $6 \mathrm{~km} \mathrm{~s}^{-1}$.

For outflow (b), a driving source could not be identified in the IRAC images, most likely the source is deeply embedded and not visible at $8 \mu \mathrm{m}$. For source (c), however, we could identify a possible driving source. About $3^{\prime \prime}$ southeast of the $\mathrm{H}_{2}$ emission, 

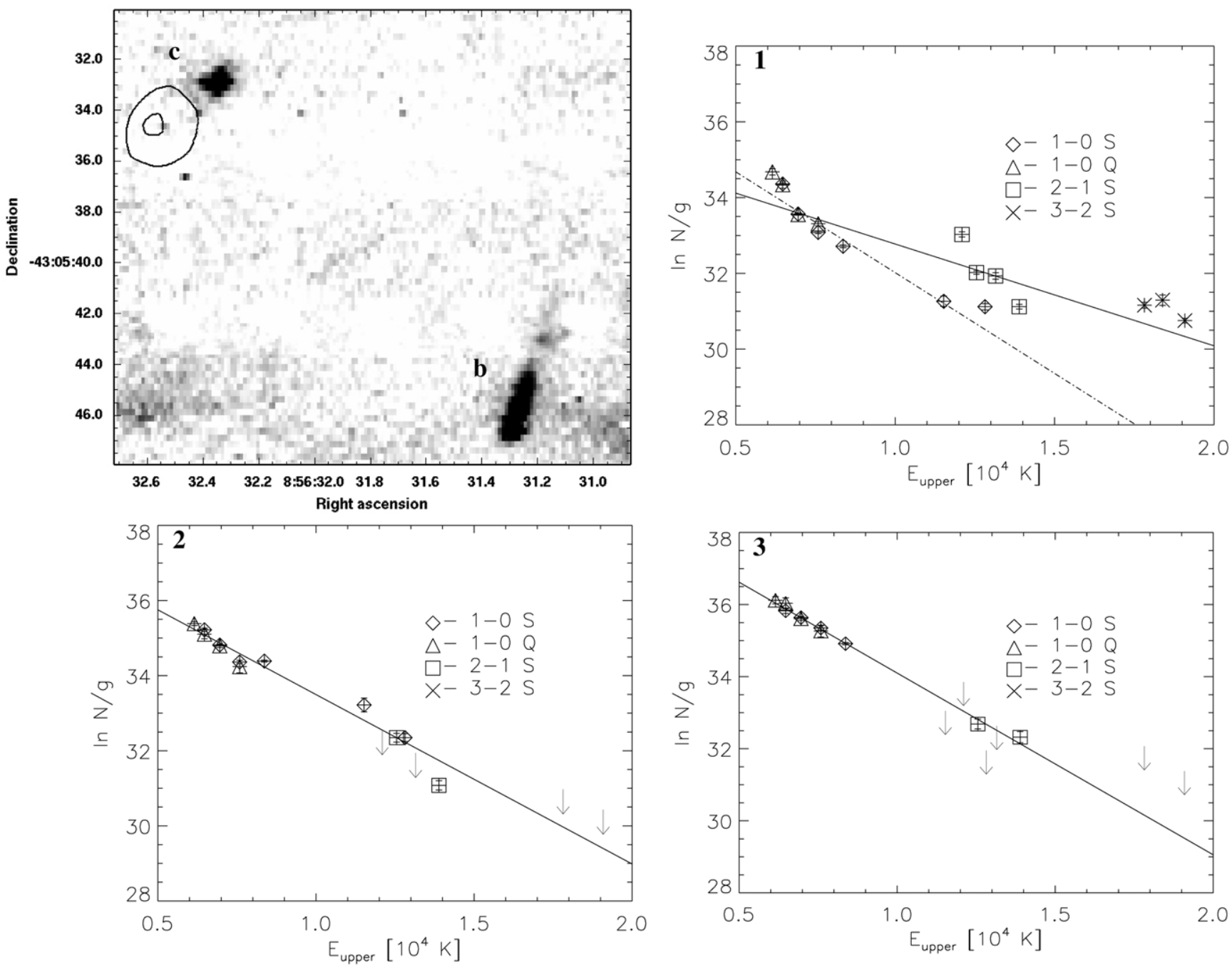

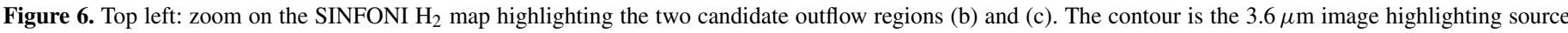

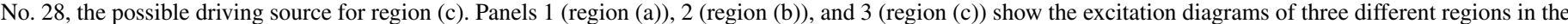

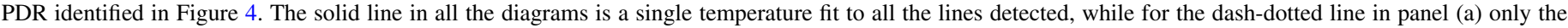

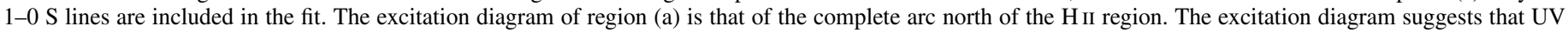
fluorescence is the excitation mechanism in this area. Regions (b) and (c) are most likely outflows as their excitation diagrams suggest thermal excitation.

a deeply embedded protostar is identified on the IRAC images with a very bright $4.5 \mu \mathrm{m}$ flux (source No. 28 in Figure 2). The location of the source is shown in Figures 4 and 6 where the $3.6 \mu \mathrm{m}$ contours of source No. 28 are overlaid on the SINFONI images. The excess $4.5 \mu \mathrm{m}$ emission is most likely coming from $\mathrm{H}_{2}$ emission closer to the source, too deeply embedded to detect at near-infrared wavelengths.

\subsection{Summary}

Summarizing, RCW 34 consists of three distinct regions. A large bubble is detected in the IRAC images, with a cluster of class II stars inside. At the northern edge of this bubble the $\mathrm{H}$ II region is located, ionized by vdBH $25 \mathrm{a}(\mathrm{O} 8.5 \mathrm{~V})$. The detected north-south extinction gradient as well as the $\mathrm{H} \alpha$ velocity gradient found by Heydari-Malayeri (1988) suggest that the $\mathrm{H}$ II region is a champagne flow.

In between the molecular cloud and the HII region, a PDR is located traced by $\mathrm{H}_{2}$ emission and $8 \mu \mathrm{m}$ emission. The star formation inside the molecular cloud seems to be more recent as masers, class 0/I sources and outflows are present in that area.

\section{SINFONI NEAR-INFRARED SPECTROSCOPY}

This section focuses on the stellar content of the NIR cluster inside the $\mathrm{H}$ II region $\mathrm{RCW} 34$. In total, 134 stars have been identified between $K=8.7$ and $17 \mathrm{mag}$ (see Figure 4 for their location). Our SINFONI observations provide an $\mathrm{H}$ - and $\mathrm{K}$ band spectrum of each source with a spectral resolution of $R=1500$. Stars with $K<14 \mathrm{mag}$ have high enough $\mathrm{S} / \mathrm{N}$ spectra to obtain a reliable spectral type. This reduces the sample to 26 stars, which will be discussed in the remainder of this paper.

To estimate the stellar content, Figure 7 shows the $K$ versus $(J-K)$ color-magnitude diagram (CMD) of the area in RCW 34 covered with SINFONI taken from Bik (2004), who imaged a $5^{\prime} \times 5^{\prime}$ field using SOFI at the NTT telescope.

The symbols in the plot represent the different spectral types derived later in this section. The CMD shows that the cluster consists of stars showing a large range in $J-K$ colors, which could be related to large-extinction variations in the cluster. In the remainder of the paper, the brightest star ( $\mathrm{vdBH} 25 \mathrm{a})$ is labeled No. 1 and the label number increases with $K$-band magnitude. Table 6 provides the list of the 26 brightest stars, 
Table 3

Equivalent Widths and $K$-band Spectral Types of the OB Stars in RCW 34

\begin{tabular}{lcccccc}
\hline \hline Star & Br11 $(1.68 \mu \mathrm{m})$ & He I $(1.70 \mu \mathrm{m})$ & He I $(2.11 \mu \mathrm{m})$ & $\mathrm{Br} \gamma(2.166 \mu \mathrm{m})$ & K-Spectral Type & Optical Spectral Type \\
\hline 1 & $2.05 \pm 0.3 \AA$ & $0.91 \pm 0.2 \AA$ & $0.4 \pm 0.4 \AA$ & $4.43 \pm 0.4 \AA$ & kO9-B1 & O8V-B1V \\
2 & $2.90 \pm 0.4 \AA$ & $0.75 \pm 0.2 \AA$ & $0.6 \pm 0.2 \AA$ & $6.07 \pm 0.3 \AA$ & kB2-B3 & B1V-B2.5V \\
3 & $5.79 \pm 0.7 \AA$ & $0.76 \pm 0.2 \AA$ & $<0.4$ & $6.06 \pm 0.4 \AA$ & kB4-B7 & B3V-B7V \\
\hline
\end{tabular}

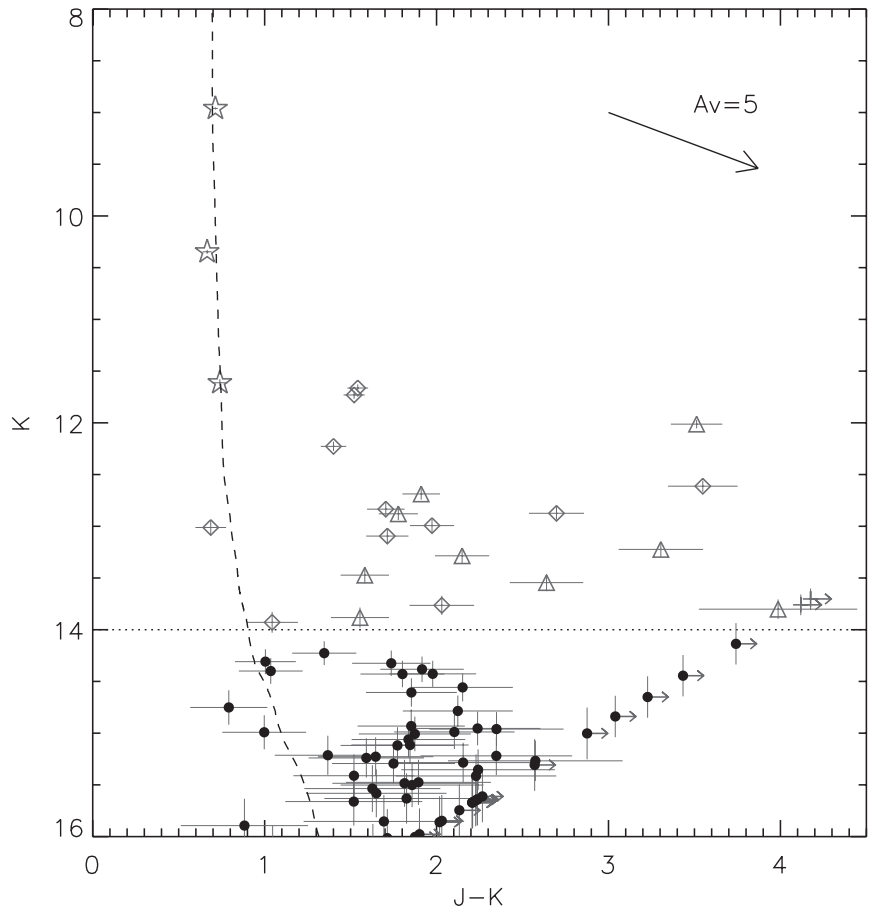

Figure 7. Observed $K$ vs. $(J-K)$ color-magnitude diagram of the region in RCW 34 covered by SINFONI taken from Bik (2004). Stars brighter than $K=14$ mag (horizontal dotted line) are marked with symbols representing their spectral types derived in Sections 4.1 and 4.2 (star symbols: OB stars, diamonds: $\mathrm{G}$ stars, triangles: $\mathrm{K}$ stars). The featureless stars are displayed as crosses. The dashed line represents the $2 \mathrm{Myr}$ isochrone taken from Lejeune \& Schaerer (2001).

as well as some additional objects of special interest, and their properties derived in this paper.

\subsection{Early-type Stars}

The SINFONI H+K spectra of the three brightest cluster members (sources No. 1, No. 2, and No. 3) show Br $\gamma$ absorption in the $K$ band and $\mathrm{Br} 10-14$ absorption in the $H$ band (Figure 8 ). Weaker lines of He I are also visible in $H(1.70 \mu \mathrm{m})$ and $K(2.058$ and $2.11 \mu \mathrm{m}$ ). To obtain a spectral type estimate, we apply the $K$-band classification scheme for OB stars from Hanson et al. (1996). Their classification links a $K$-band spectral type to an optical spectral type. For late $\mathrm{O}$ and early $\mathrm{B}$ stars, the classification is based on $\mathrm{He} \mathrm{I}(2.11 \mu \mathrm{m})$ and $\mathrm{Br} \gamma$. For the $H$ band, we used the classification of Hanson et al. (1998) and Blum et al. (1997) where the important lines are He I $(1.700 \mu \mathrm{m})$ and $\operatorname{Br} 11(1.68 \mu \mathrm{m})$.

Table 3 shows the measured equivalent widths (EW) of the relevant lines in the $H$ - and $K$-band spectra of the three OB stars. In Column 6 , the $K$-band spectral type is given, with the corresponding optical spectral type listed in Column 7. The brightest star, No. 1, has a spectral type between O8V and B1V based on the presence of He I $(2.11 \mu \mathrm{m})$ and moderately strong $\mathrm{Br} \gamma$. Star No. 2 has a much stronger $\mathrm{Br} \gamma$ absorption and is
Table 4

Properties of the Detected OB Stars in RCW 34

\begin{tabular}{lcrcc}
\hline \hline Star & Spectral Type & \multicolumn{1}{c}{$K(\mathrm{mag})$} & $J-K(\mathrm{mag})$ & $A_{V}(\mathrm{mag})$ \\
\hline 1 & O8.5V & $8.96 \pm 0.01$ & $0.73 \pm 0.01$ & $4.2 \pm 0.1$ \\
2 & B0.5V-B1V & $10.35 \pm 0.02$ & $0.68 \pm 0.03$ & $3.9 \pm 0.2$ \\
3 & B2V-B3V & $11.61 \pm 0.03$ & $0.75 \pm 0.05$ & $4.1 \pm 0.4$ \\
\hline
\end{tabular}

therefore classified as B1V-B2.5V. The fainter OB star (No. 3) does not have He I $(2.11 \mu \mathrm{m})$ in its spectrum and is therefore classified as B3V-B7V. The EWs of the $H$-band lines are in agreement with the $K$-band spectral type. The only deviating value is the very strong Br11 absorption of No. 3, which is about equally strong as the $\mathrm{Br} \gamma$ line. The strength of $\mathrm{Br} 11$ is compatible with that of a supergiant.

The spectral type of star, No. 1, (O8V-B1V) is compatible with the optical classification as a $08.5 \mathrm{~V}$ star by HeydariMalayeri (1988). As the optical classification is more reliable than our near-IR classification, we will adopt a spectral type of O8.5V for No. 1 as the main ionizing star for RCW 34. This spectral type is in agreement with the Lyman continuum flux derived from radio continuum data (Caswell \& Haynes 1987), and is also compatible with the $\mathrm{He} \mathrm{I}$ and $\mathrm{Br} \gamma$ emission of the $\mathrm{H}$ II region (Section 3.2.1). This corresponds to a mass of $20 M_{\odot}$ for star No. 1 (Martins et al. 2005).

The color of early-type stars is a good measure of the extinction. For the intrinsic colors, we used the values given by Koornneef (1983). We used the extinction law of Cardelli et al. (1989); the derived extinction toward the three stars is listed in Table 4 and ranges from $A_{V}=3.9$ to $4.2 \mathrm{mag}$ ( $A_{K}=0.42$ to $0.45 \mathrm{mag}$ ). The error in the $A_{V}$ values reflects both the photometric errors and the uncertainties in $(J-K)_{0}$ due to uncertainties in their spectral classification. These values are in good agreement with those derived from the $\mathrm{Br} 10 / \mathrm{Br} \gamma$ ratio of the H II spectra (Section 3.2.1).

The spectral types and extinctions of the OB stars, coupled with their absolute magnitudes, allow a distance determination. For the O8.5V star No. 1, we obtain the absolute magnitude from Martins \& Plez (2006) and Aller et al. (1982). This results in a distance of $2500 \pm 200 \mathrm{pc}$ for the $08.5 \mathrm{~V}$ star. The uncertainty reflects the difference in the absolute magnitudes between Martins \& Plez (2006) and Aller et al. (1982). Hanson et al. (1997) and Blum et al. (2000) provide absolute magnitudes for zero-age main sequence (ZAMS) stars. Their results are about 1 mag fainter than corresponding values for the main sequence of the Hertzsprung-Russell diagram (HRD). If we adopt the ZAMS magnitudes, we obtain a distance of $1800 \pm 200 \mathrm{pc}$. The cluster age is probably a few megayears, hence we prefer the main-sequence values. Thus, we adopt $2500 \pm 200 \mathrm{pc}$ as the distance to RCW 34. This distance is slightly closer than the $2.9 \mathrm{kpc}$ derived by Heydari-Malayeri (1988) using the same spectral type and optical magnitudes. The difference results from the different absolute magnitudes and extinction we apply.

Using the $2.5 \mathrm{kpc}$ distance, we can refine the spectral type classification of the two B stars in the cluster. Using the distance 

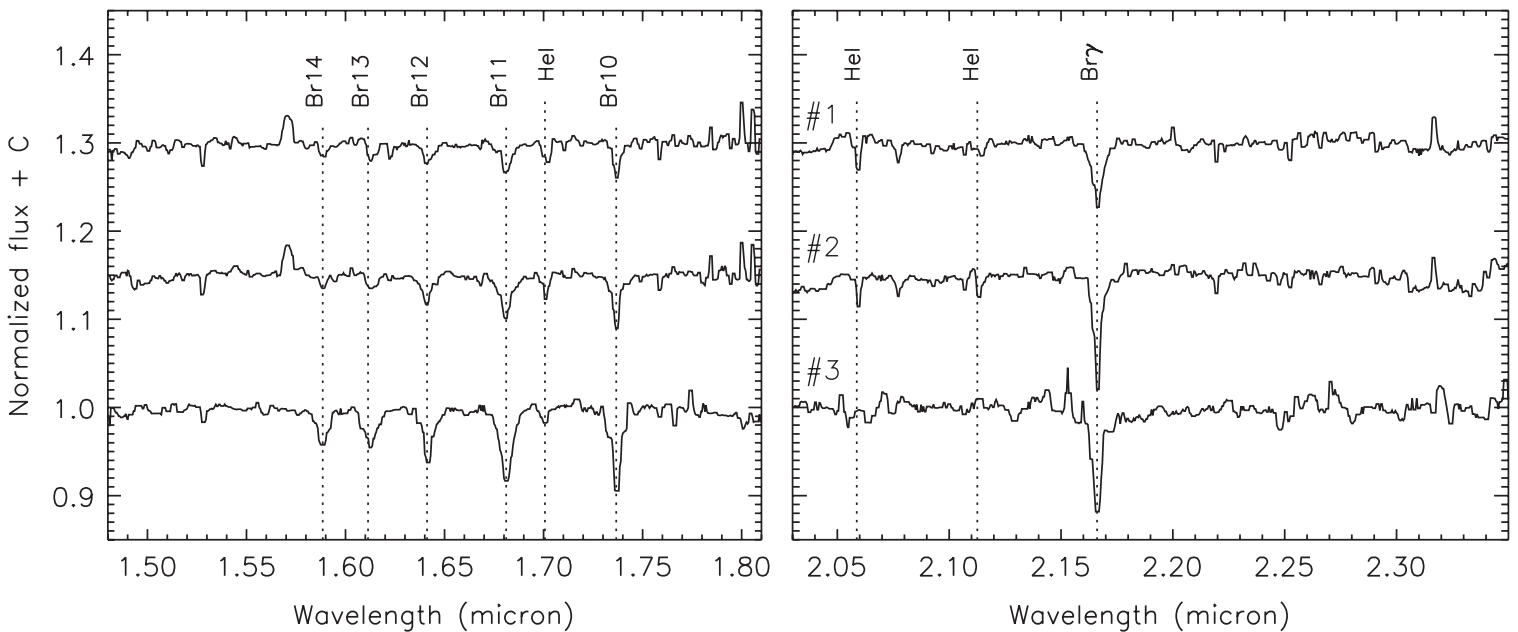

Figure 8. Normalized SINFONI $H$-band (left panel) and $K$-band spectra of the three OB stars detected in RCW 34 . Their spectra are characterized by absorption lines of the hydrogen Brackett series and He I (dashed vertical lines).

modulus, the $A_{V}$, and the observed brightness, we derive an absolute $K$-band magnitude of $M(K)=-2.1 \mathrm{mag}$ for No. 2 and $M(K)=-0.8 \mathrm{mag}$ for No. 3. With the calibration of Aller et al. (1982) this results in a B0.5-B1 V spectral type for No. 2 and a B2-B3V spectral type for No. 3 (Table 4), still compatible with the original near-infrared spectroscopic classification. These spectral types correspond to a mass of 11 and $7 M_{\odot}$, respectively (Aller et al. 1982).

\subsection{Late-type Stars}

Besides the 3 OB stars, we found 21 stars showing absorption lines typical of later spectral type (Figure 9). The most prominent lines are the $\mathrm{CO}$ first overtone absorption bands between 2.29 and $2.45 \mu \mathrm{m}$, and absorption lines of $\mathrm{Mg}_{\mathrm{I}}$ and other atomic species in the $H$ band as well as $\mathrm{Ca}$ I and $\mathrm{Na}$ I in the $K$ band. To determine if these stars are cluster members, we need to obtain their spectral type and luminosity class.

For the classification of the late-type stars, we use the reference spectra of Cushing et al. (2005) and Rayner et al. (2009). These spectra are taken with the IRTF telescope ${ }^{15}$ and cover the spectral types between $\mathrm{F}$ and $\mathrm{M}$ in all luminosity classes with a resolution between $R=2000$ and 5000. These reference spectra were convolved and rebinned to the resolution of SINFONI. We corrected for the differences in spectral slope due to the extinction of our SINFONI targets using the extinction law of Cardelli et al. (1989). The atomic lines, like Mg I and $\mathrm{Na} \mathrm{I}$, are used for the temperature determination, while the $\mathrm{CO}$ and $\mathrm{OH}$ lines serve as a luminosity indicator.

Comparison of the SINFONI spectra with reference spectra indicates that the CO $(2.29 \mu \mathrm{m})$ absorption is usually deeper than in dwarf reference spectra, but not as deep as in the giant spectra. In the few cases where a luminosity class IV reference spectrum was available, the spectrum provided a better match to the observed spectrum. This suggests that our late-type stars are low- and intermediate-mass pre-main-sequence (PMS) stars; indeed, Luhman (1999) and Winston et al. (2009) find that PMS spectra have a surface gravity intermediate between giant and dwarf spectra. If the stars were giants, dust veiling could make the $K$-band $\mathrm{CO}$ lines weaker. However, the $H$-band $\mathrm{CO}$ and $\mathrm{OH}$ lines are also much weaker, while the atomic lines have the

\footnotetext{
15 http://irtfweb.ifa.hawaii.edu/ spex/WebLibrary/index.html
}

expected EWs. Therefore, this seems to be a surface gravity effect, suggesting a PMS nature of our late-type stars.

We used the relation from Kenyon \& Hartmann (1995) to convert the spectral type into effective temperature. This relation applies only for main-sequence stars; for PMS stars, a different relation may hold (Hillenbrand 1997; Winston et al. 2009). Cohen \& Kuhi (1979) show that the temperature of PMS stars might be overestimated by values between $500 \mathrm{~K}$ (G stars) and $200 \mathrm{~K}$ (mid-K). We took this source of error into account when calculating the errors in the effective temperature.

Table 5 shows the result of the classification for all the latetype stars brighter than $K=14 \mathrm{mag}$. The spectral types range from early $\mathrm{G}$ to mid-K; about half of the stars have a surface gravity indicative of a PMS nature, their luminosity class is marked as "PMS." The stars classified as dwarfs are candidate foreground stars, but this does not exclude them as PMS cluster members, as Winston et al. (2009) also find PMS stars with surface gravity very close to the dwarf values.

With the knowledge of the spectral type, the observed color can be converted into an extinction estimate. The intrinsic $J-K$ color for dwarfs and giants can differ as much as $0.4 \mathrm{mag}$ for late K stars (Koornneef 1983). As the late-type stars are PMS stars, we used the mean of the color for dwarfs and giants as the intrinsic $J-K$ color. The difference between the mean value and the dwarf and giant values is used as the error in the intrinsic color. The derived extinctions are listed in Table 6. Source No. 17 does not have a $J$-band magnitude as the photometry is too contaminated by source No. 1 ; it is excluded from further analysis.

All the stars with $A_{V}>12 \mathrm{mag}$ (No. 6, No. 8, No. 16, and No. 24) are located north of the $\mathrm{Br} \gamma$ emission in the region where the $\mathrm{H}_{2}$ and IRAC dust emission is present. Their extinction is consistent with being embedded in the molecular cloud north of the $\mathrm{H}$ II region. The objects situated amidst the $\mathrm{Br} \gamma$ emission show a visual extinction between 4 and 10 mag, consistent with that measured from the nebular line spectrum (Section 3.2.1).

To confirm the PMS nature of the objects, we looked for $\mathrm{Br} \gamma$ emission from the source. We found two point sources in the continuum subtracted $\mathrm{Br} \gamma$ line map. One of the sources corresponds to No. 23, a G8 PMS star. Following Muzerolle et al. (1998), this $\mathrm{Br} \gamma$ emission could arise from accreting matter. The other $\mathrm{Br} \gamma$ emitting point source is No. 27 (Table 6). This star is rather faint $(K=15.5 \pm 0.1 \mathrm{mag})$ and the continuum has too 

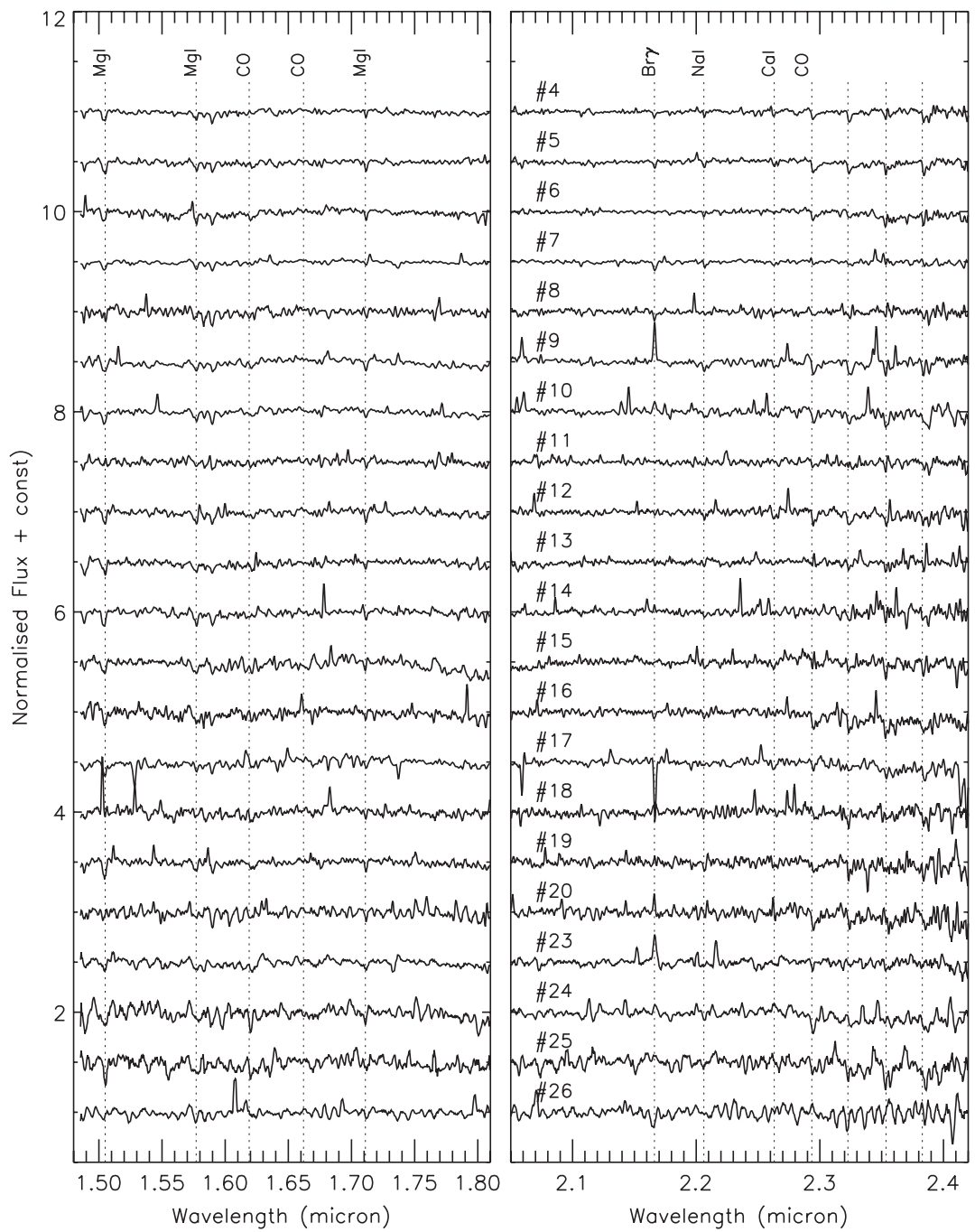

Figure 9. Normalized SINFONI $H$-band (left panel) and $K$-band (right panel) spectra of the 21 objects showing late-type stellar spectra. Most of these objects are PMS stars. The vertical dashed lines show the location of some of the absorption lines used for the classification of the stars. The stars are numbered according to Table 6 .

low $\mathrm{S} / \mathrm{N}$ to detect any absorption features. The emission line spectrum also shows He I $(2.058 \mu \mathrm{m})$ and the Brackett lines in the $H$ band, identical to the surrounding $\mathrm{H}$ II region (Figure 5). The $\mathrm{Br} \gamma$ emission from this object could arise from a disk being photo-evaporated by the main ionizing star. These "proplyds" have been found in Orion (O'Dell 2001) and other high-mass star-forming regions like M8 (Stecklum et al. 1998) and RCW 38 (DeRose et al. 2009). The spatial resolution of our data $\left(\sim 0^{\prime \prime} 6,1500 \mathrm{AU}\right.$ at $\left.2.5 \mathrm{kpc}\right)$ did not allow us to resolve the proplyd, which is consistent with the sizes (up to $500 \mathrm{AU}$ ) measured for the Orion Proplyds (O'Dell 1998). The observed $K$-band magnitude and lower limit of source No. 27 does seem consistent with near-infrared observations of the Orion Proplyds (Rost et al. 2008).

In the spectra of, e.g., source No. 9 and No. 17, emission and absorption of $\mathrm{Br} \gamma$ is detected as well. These lines are artifacts from the $\mathrm{Br} \gamma$ removal using the median filtering. In the $\mathrm{Br} \gamma$ line image, no extra emission or absorption is present.

Two objects brighter than $K=14$ mag are unclassified. These objects (No. 21 and No. 22) have very red spectra and a rather low $\mathrm{S} / \mathrm{N}$. We could not identify any features in their spectra. As the spectra are extremely red, they are likely dominated by dust emission and therefore the spectrum of the underlying star might not be visible. Like the late-type stars with high extinction, these featureless spectra are located in the high-extinction area, in the north of the SINFONI field and therefore are most likely embedded stars.

The remaining sources are too faint to obtain a proper classification. In the spectra of some of these objects, CO absorption at $2.29 \mu \mathrm{m}$ can still be detected, but the spectra are of too low $\mathrm{S} / \mathrm{N}$ to detect the atomic lines needed for an effective temperature classification. A handful of the objects have a $J-K$ color around $1.0 \mathrm{mag}$ (see Figure 7), indicating that these might be late-type foreground stars with very little extinction. Most of the faint objects are concentrated around $J-K=2.0 \mathrm{mag}$. This color is similar to that of the brighter PMS stars around $A_{V}=$ 5-10 mag. The redder and more deeply embedded objects are not observable as they are too faint to detect in the $J$ band. The objects with $K>14 \mathrm{mag}$, clustered around $J-K=2 \mathrm{mag}$, are most likely PMS stars with masses below $1.5-2 M_{\odot}$.

\subsection{IR Excess}

In total, 17 sources (out of 26) have been detected in one or more Spitzer/IRAC bands with only three sources detected in all four bands. Most of the sources are only detected at 3.6 and $4.5 \mu \mathrm{m}$. At longer wavelengths, the high and variable background emission makes the detection of point sources very difficult. The objects detected in all four bands are labeled in Figure 2. One of the PMS stars (No. 11) is identified as a class II 
Table 5

$T_{\text {eff }}$, Spectral Type, Luminosity Class, and IR Excess of the Detected Late-type Stars Brighter than $K=14$ mag

\begin{tabular}{|c|c|c|c|c|}
\hline Star & $T_{\text {eff }}(\mathrm{K})$ & Sp. Type & Lum. Class & IR Excess \\
\hline 4 & $5520 \pm 365$ & $\mathrm{G} 8 \pm 1$ & PMS & - \\
\hline 5 & $5410 \pm 365$ & $\mathrm{G} 9 \pm 1$ & PMS & $\mathrm{n}$ \\
\hline 6 & $5080 \pm 380$ & $\mathrm{~K} 1 \pm 1$ & PMS & + \\
\hline 7 & $5860 \pm 415$ & $\mathrm{G} 2 \pm 2$ & V & + \\
\hline 8 & $5680 \pm 360$ & G6.5 \pm 1 & V & - \\
\hline 9 & $4900 \pm 350$ & $\mathrm{~K} 2 \pm 1$ & PMS & + \\
\hline 10 & $5520 \pm 355$ & $\mathrm{G} 8 \pm 1$ & PMS & - \\
\hline 11 & $5800 \pm 405$ & $\mathrm{G} 4 \pm 1$ & PMS & + \\
\hline 12 & $4900 \pm 445$ & $\mathrm{~K} 2 \pm 2$ & PMS & $\mathrm{n}$ \\
\hline 13 & $5680 \pm 360$ & $\mathrm{G} 6.5 \pm 1$ & PMS & - \\
\hline 14 & $5680 \pm 360$ & G6.5 \pm 1 & $\mathrm{~V}$ & + \\
\hline 15 & $5520 \pm 355$ & $\mathrm{G} 8 \pm 1$ & V & - \\
\hline 16 & $5080 \pm 380$ & $\mathrm{~K} 1 \pm 1$ & PMS & $\mathrm{n}$ \\
\hline 17 & $5080 \pm 380$ & $\mathrm{~K} 1 \pm 1$ & PMS & $\mathrm{n}$ \\
\hline 18 & $5080 \pm 380$ & $\mathrm{~K} 1 \pm 1$ & $\mathrm{~V}$ & + \\
\hline 19 & $4900 \pm 350$ & $\mathrm{~K} 2 \pm 1$ & $\mathrm{~V}$ & + \\
\hline 20 & $4900 \pm 350$ & $\mathrm{~K} 2 \pm 1$ & PMS & $\mathrm{n}$ \\
\hline 23 & $5520 \pm 355$ & $\mathrm{G} 8 \pm 1$ & PMS & + \\
\hline 24 & $4800 \pm 515$ & Early K & $?$ & $\mathrm{n}$ \\
\hline 25 & $4800 \pm 515$ & Early K & $?$ & + \\
\hline 26 & $5410 \pm 365$ & $\mathrm{G} 9 \pm 1$ & $\mathrm{~V}$ & $\mathrm{n}$ \\
\hline
\end{tabular}

Note. "+": infrared excess is present, "-" no infrared excess detected, "n": infrared excess could not be determined due to bright background emission.

object. The other two objects (No. 14 and No. 21) are classified as class 0/I and will be discussed in Section 5.3.

To determine if the PMS stars have an infrared excess, we construct the spectral energy distribution (SED) and compare it with low-resolution Kurucz spectra (Kurucz 1993) for the $1-8 \mu \mathrm{m}$ range. This allows us to determine the IR excess even for objects detected in only 1 or 2 IRAC bands. The Column 5 of Table 5 shows that nine PMS stars show a detectable IR excess (marked as a "+"), while for five sources no IR excess is detected (marked as a "-"). The remaining sources are marked with an "n": no IRAC counterpart could be detected due to blending with other sources or spatial coincidence with the bright, extended arc emission. The infrared excess is already present at $3.6 \mu \mathrm{m}$ with an excess of more than a magnitude in some cases. The detected IR excess of at least nine of the PMS star candidates reinforces the hypothesis that these stars are young and still surrounded by a circumstellar disk.

The SINFONI spectra, however, suggest that the near-infrared excess of most PMS stars is low, as all these stars have absorption lines in their $K$-band spectra. Should a strong IR excess be present these lines would not have been detected. The $K$-band spectrum of No. 11 suggests that some veiling might be present; the $\mathrm{CO}$ was still stronger than that of the best-fitting dwarf reference spectrum, but the $\mathrm{NaI}$ and $\mathrm{Ca}$ I lines in the $K$ band were very faint. In the spectra of the two sources where no absorption features have been detected (No. 21 and No. 22), a NIR excess is most likely present. These sources also have very red colors and a strong IRAC excess.

\section{STELLAR POPULATION}

\subsection{Cluster Membership}

To discuss the stellar content in detail, we first need to separate the cluster members from the fore- and background stars. The O8.5V star (source No. 1) ionizes the $\mathrm{HII}$ region and is also responsible for the zone of interaction with the molecular cloud to the north. Analysis of the brightness and spectral type of the B stars (sources No. 2 and No. 3) shows that they are located at the same distance $(2.5 \mathrm{kpc})$ and have a similar extinction as the main ionizing source and therefore are considered to be cluster members.

It is less obvious that all the late-type stars are cluster members as well. As discussed in Section 4.2, most of the late-type stars have a surface gravity typical for PMS stars. Additionally, about half of the stars have an IR excess, supporting the result from the spectroscopy that those stars are PMS stars surrounded by an accretion disk. The extinction derived for these stars is consistent with the extinction derived using the $\mathrm{H}_{\mathrm{II}}$ region and the $\mathrm{H}_{2}$ emission.

Sources No. 13 and No. 15 show no evidence for a PMS nature or for an IR excess, but have higher visual extinctions (5.6 and 7 mag, respectively) than observed from the $\mathrm{H}$ II emission in that area. Their early spectral types makes it difficult to derive a luminosity class because of the $\mathrm{CO}$ bands are not present. The high extinction suggests that they are background stars, but then the stars should be (super) giants as dwarfs are too faint to be seen as background stars.

Two more stars have $A_{V}$ values inconsistent with their location: sources No. 14 and No. 26 show very low extinction ( 0.25 and $1.8 \mathrm{mag}$, respectively) while they are located toward the high-extinction area north of the H II region. Star No. 14 has virtually no extinction and a surface gravity typical for main-sequence stars, however, it coincides with an IRAC source having colors of a class $0 / \mathrm{I}$ object. Also, on the $\mathrm{Br} \gamma$ line map some faint $\mathrm{Br} \gamma$ emission is seen surrounding the star. The fact that this star has no extinction is inconsistent with its IRAC colors and a protostar nature. If the star were a protostar, it would be more deeply embedded and have a much larger $A_{V}$. It is most likely a chance alignment of a foreground late $\mathrm{G}$ star (as seen in the NIR) with a deeply embedded protostar only seen at mid-IR wavelengths. Additionally, star No. $26\left(A_{V}=1.8 \mathrm{mag}\right)$, also located toward the high-extinction area, does not show any PMS characteristic nor an IR excess and also is most likely a foreground star. The locations of the stars in the SINFONI field are shown in Figure 10. The different classes of objects do not cover a preferred area of the SINFONI field, but rather are spread across the entire field.

Summarizing, after analysis of their location, reddening and spectral features, we conclude that most of the stars are probably cluster members, with two exceptions where the stars are probably foreground stars (No. 14 and No. 26). The nature of sources No. 13 and No. 15 is unclear, but their high extinction suggests that they are related to the cluster.

\subsection{Stellar Content and Age}

To constrain the mass of the PMS stars and the age of RCW 34, we construct an HRD to compare the observed parameters with PMS evolutionary tracks. The derived extinctions allow us to de-redden the $K$-band magnitude, convert to absolute magnitude with the derived temperature, and plot the points in a $K$ versus $\log \left(T_{\text {eff }}\right)$ diagram (Figure 11, left panel). We exclude the foreground stars No. 14 and No. 26 to enable a comparison with the isochrones. The symbols reflect the different spectral types. Filled symbols indicate that a Spitzer/ IRAC excess emission was detected.

Overplotted as a dotted line is the 2 Myr main-sequence isochrone taken from Lejeune \& Schaerer (2001). The $2 \mathrm{Myr}$ isochrone is chosen to illustrate the main sequence; $1 \mathrm{Myr}$ or 
Table 6

Photometric and Spectroscopic Properties of the Sources Located in the SINFONI Field of View

\begin{tabular}{|c|c|c|c|c|c|c|c|c|c|c|c|}
\hline Star & $\begin{array}{l}\text { R.A. (2000) } \\
\quad(\mathrm{h} \mathrm{m} \mathrm{s})\end{array}$ & $\begin{array}{c}\text { Decl. (2000) } \\
\left(0^{\prime} \prime^{\prime \prime}\right)\end{array}$ & $\begin{array}{c}K \\
(\mathrm{mag})\end{array}$ & $\begin{array}{c}J-K \\
(\mathrm{mag})\end{array}$ & $\begin{array}{c}{[3.6]} \\
(\mathrm{mag})\end{array}$ & $\begin{array}{l}{[4.5]} \\
(\mathrm{mag})\end{array}$ & $\begin{array}{c}{[5.8]} \\
(\mathrm{mag})\end{array}$ & $\begin{array}{c}{[8.0]} \\
(\mathrm{mag})\end{array}$ & Sp. Type & $\begin{array}{c}A_{V} \\
(\mathrm{mag})\end{array}$ & Comments \\
\hline 1 & $08: 56: 28.1$ & $-43: 05: 55.6$ & $8.96 \pm 0.01$ & $0.73 \pm 0.01$ & $\ldots$ & $\ldots$ & $\ldots$ & $\ldots$ & $08.5 \mathrm{~V}$ & $4.8 \pm 0.1$ & $\mathrm{vdBH} 25 \mathrm{a}$ \\
\hline 2 & $08: 56: 28.1$ & $-43: 05: 57.1$ & $10.35 \pm 0.02$ & $0.69 \pm 0.03$ & $\ldots$ & $\ldots$ & $\ldots$ & $\ldots$ & B0.5-B1V & $4.1 \pm 0.2$ & \\
\hline 3 & $08: 56: 29.8$ & $-43: 05: 59.4$ & $11.61 \pm 0.03$ & $0.76 \pm 0.05$ & $10.81 \pm 0.03$ & $10.37 \pm 0.06$ & $\ldots$ & $\ldots$ & B2-B3V & $4.3 \pm 0.4$ & \\
\hline 4 & $08: 56: 31.7$ & $-43: 05: 56.3$ & $11.66 \pm 0.04$ & $1.56 \pm 0.06$ & $11.26 \pm 0.03$ & $11.01 \pm 0.06$ & $\ldots$ & $\ldots$ & $\mathrm{G} 8 \pm 1 \mathrm{PMS}$ & $4.7 \pm 0.6$ & \\
\hline 5 & $08: 56: 28.2$ & $-43: 05: 58.3$ & $11.73 \pm 0.04$ & $1.54 \pm 0.06$ & $\ldots$ & $\ldots$ & $\ldots$ & $\ldots$ & $\mathrm{G} 9 \pm 1 \mathrm{PMS}$ & $4.6 \pm 0.6$ & \\
\hline 6 & 08:56:29.1 & $-43: 05: 31.6$ & $12.01 \pm 0.04$ & $3.53 \pm 0.15$ & $9.67 \pm 0.02$ & $\ldots$ & $8.51 \pm 0.16$ & $\ldots$ & $\mathrm{K} 1 \pm 1 \mathrm{PMS}$ & $14.2 \pm 0.9$ & \\
\hline 7 & $08: 56: 29.6$ & $-43: 05: 51.9$ & $12.23 \pm 0.05$ & $1.42 \pm 0.07$ & $10.84 \pm 0.17$ & $\ldots$ & $\ldots$ & $\ldots$ & $\mathrm{G} 2 \pm 2 \mathrm{~V}$ & $4.4 \pm 0.6$ & \\
\hline 8 & 08:56:31.0 & $-43: 05: 32.4$ & $12.61 \pm 0.05$ & $3.6 \pm 0.2$ & $11.61 \pm 0.07$ & & $\ldots$ & $\ldots$ & $\mathrm{G} 6.5 \pm 1 \mathrm{~V}$ & $15.0 \pm 1.3$ & \\
\hline 9 & 08:56:29.7 & $-43: 06: 03.2$ & $12.68 \pm 0.06$ & $1.93 \pm 0.11$ & $10.88 \pm 0.03$ & $10.49 \pm 0.04$ & $9.7 \pm 0.10$ & $\ldots$ & K2 \pm 1 PMS $/ \mathrm{V}$ & $5.7 \pm 0.7$ & \\
\hline 10 & 08:56:29.7 & $-43: 06: 24.3$ & $12.83 \pm 0.06$ & $1.72 \pm 0.11$ & $12.17 \pm 0.03$ & $11.88 \pm 0.05$ & $\ldots$ & $\ldots$ & G8 \pm 1 PMS & $5.5 \pm 0.8$ & \\
\hline 11 & 08:56:31.9 & $-43: 06: 01.5$ & $12.87 \pm 0.06$ & $2.71 \pm 0.16$ & $11.00 \pm 0.02$ & $10.36 \pm 0.02$ & $9.76 \pm 0.04$ & $8.92 \pm 0.08$ & $\mathrm{G} 4 \pm 1 \mathrm{PMS} / \mathrm{V}$ & $10.8 \pm 1.1$ & Class II \\
\hline 12 & $08: 56: 29.1$ & $-43: 06: 19.1$ & $12.88 \pm 0.06$ & $1.80 \pm 0.11$ & $\ldots$ & 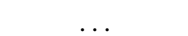 & $\ldots$ & $\ldots$ & $\mathrm{K} 2 \pm 2 \mathrm{PMS} / \mathrm{V}$ & $5.0 \pm 0.7$ & \\
\hline 13 & $08: 56: 27.8$ & $-43: 06: 21.4$ & $12.99 \pm 0.06$ & $1.99 \pm 0.13$ & $12.17 \pm 0.04$ & $11.67 \pm 0.07$ & $\ldots$ & $\ldots$ & $\mathrm{G} 6.5 \pm 1 \mathrm{~V}$ & $7.0 \pm 0.9$ & \\
\hline 14 & $08: 56: 26.8$ & $-43: 05: 35.2$ & $13.01 \pm 0.07$ & $0.71 \pm 0.09$ & $10.51 \pm 0.12$ & $9.89 \pm 0.10$ & $7.10 \pm 0.11$ & $5.21 \pm 0.11$ & $\mathrm{G} 6.5 \pm 1 \mathrm{~V}$ & $0.3 \pm 0.7$ & $\mathrm{vdBH} 25 \mathrm{a} C$ \\
\hline 15 & $08: 56: 26.7$ & $-43: 06: 27.0$ & $13.09 \pm 0.07$ & $1.73 \pm 0.12$ & $12.38 \pm 0.04$ & $12.00 \pm 0.07$ & $\ldots$ & $\ldots$ & $\mathrm{G} 8 \pm 1 \mathrm{~V}$ & $5.6 \pm 0.9$ & \\
\hline 16 & $08: 56: 29.2$ & $-43: 05: 32.5$ & $13.22 \pm 0.07$ & $3.32 \pm 0.25$ & $\ldots$ & $\ldots$ & $\ldots$ & $\ldots$ & $\mathrm{K} 1 \pm 1 \mathrm{PMS}$ & $13.1 \pm 1.5$ & \\
\hline 17 & $08: 56: 28.0$ & $-43: 05: 55.2$ & $13.23 \pm 0.07$ & $\ldots \pm 0.08$ & $\ldots$ & $\ldots$ & $\ldots$ & $\ldots$ & $\mathrm{K} 1 \pm 1 \mathrm{PMS} / \mathrm{V}$ & $\ldots$ & \\
\hline 18 & 08:56:29.4 & $-43: 06: 00.3$ & $13.29 \pm 0.07$ & $2.17 \pm 0.16$ & $10.90 \pm 0.03$ & $10.21 \pm 0.04$ & $9.42 \pm 0.10$ & $\ldots$ & $\mathrm{K} 1 \pm 1 \mathrm{~V}$ & $7.2 \pm 1.0$ & \\
\hline 19 & $08: 56: 27.8$ & $-43: 06: 07.5$ & $13.47 \pm 0.08$ & $1.60 \pm 0.14$ & $12.01 \pm 0.10$ & $11.06 \pm 0.11$ & $\ldots$ & $\ldots$ & $\mathrm{K} 2 \pm 1 \mathrm{~V}$ & $4.0 \pm 0.9$ & \\
\hline 20 & $08: 56: 24.6$ & $-43: 06: 01.1$ & $13.54 \pm 0.08$ & $2.66 \pm 0.21$ & $\ldots$ & & $\ldots$ & $\ldots$ & $\mathrm{K} 2 \pm 1 \mathrm{PMS}$ & $9.4 \pm 1.3$ & \\
\hline 21 & $08: 56: 29.6$ & $-43: 05: 34.1$ & $13.70 \pm 0.09$ & $>4.20$ & $10.01 \pm 0.03$ & $9.03 \pm 0.03$ & $8.23 \pm 0.08$ & $6.96 \pm 0.13$ & Featureless & $\ldots$ & Class 0/I \\
\hline 22 & 08:56:30.7 & $-43: 05: 35.4$ & $13.76 \pm 0.09$ & $>4.14$ & $11.25 \pm 0.07$ & $10.53 \pm 0.06$ & $\ldots$ & $\ldots$ & Featureless & $\ldots$ & \\
\hline 23 & 08:56:28.0 & $-43: 06: 13.2$ & $13.76 \pm 0.09$ & $2.05 \pm 0.19$ & $12.40 \pm 0.11$ & $11.66 \pm 0.15$ & $\ldots$ & $\ldots$ & G8 \pm 1 PMS $/ V$ & $7.2 \pm 1.2$ & $\mathrm{Br} \gamma$ emission \\
\hline 24 & $08: 56: 28.4$ & $-43: 05: 34.9$ & $13.80 \pm 0.09$ & $4.01 \pm 0.46$ & $\ldots$ & $\ldots$ & $\ldots$ & $\ldots$ & Early K & $15.9 \pm 2.8$ & \\
\hline 25 & 08:56:29.1 & $-43: 06: 18.5$ & $13.88 \pm 0.10$ & $1.57 \pm 0.17$ & $11.75 \pm 0.04$ & $11.27 \pm 0.06$ & $10.8 \pm 0.15$ & $\ldots$ & Early K & $3.6 \pm 1.4$ & \\
\hline 26 & $08: 56: 26.8$ & $-43: 05: 40.8$ & $13.93 \pm 0.10$ & $1.06 \pm 0.15$ & $\ldots$ & $\ldots$ & $\ldots$ & $\ldots$ & $\mathrm{G} 9 \pm 1 \mathrm{~V}$ & $1.8 \pm 1.0$ & $\mathrm{vdBH} 25 \mathrm{q}$ \\
\hline 27 & 08:56:29.0 & $-43: 05: 54.0$ & $15.5 \pm 0.1$ & $>2.3$ & $\ldots$ & $\ldots$ & $\ldots$ & $\ldots$ & $\ldots$ & $\ldots$ & Proplyd \\
\hline 28 & $08: 56: 32.5$ & $-43: 05: 34.69$ & $\ldots$ & $\ldots$ & $11.69 \pm 0.03$ & $9.77 \pm 0.02$ & $8.16 \pm 0.02$ & $6.00 \pm 0.03$ & $\ldots$ & $\ldots$ & Class 0/I \\
\hline 29 & 08:56:28.0 & $-43: 05: 53.76$ & $\ldots$ & $\ldots$ & $8.24 \pm 0.01$ & $7.57 \pm 0.02$ & $6.43 \pm 0.03$ & $4.49 \pm 0.04$ & $\ldots$ & $\ldots$ & Class 0/I \\
\hline
\end{tabular}




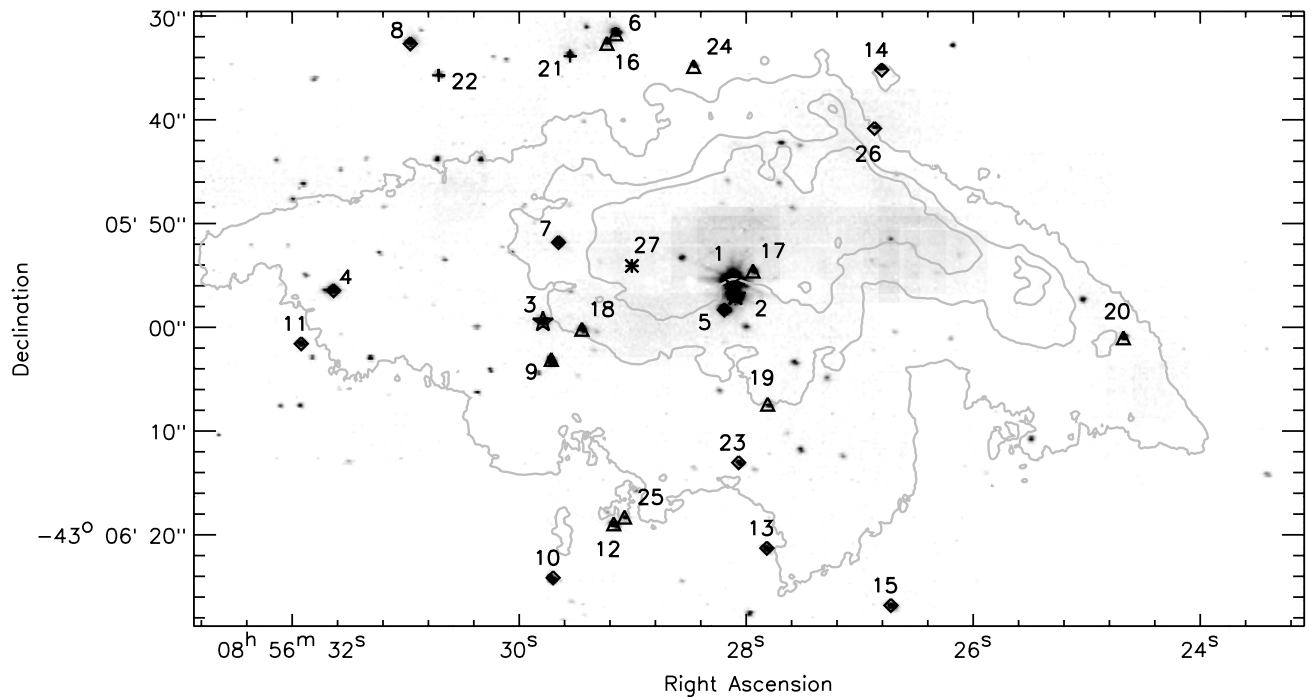

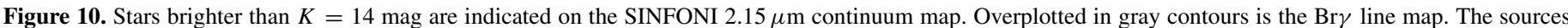

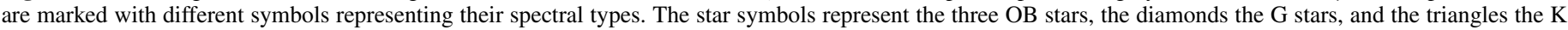
stars. The featureless stars (No. 21 and No. 22) correspond to the cross signs. The location of the proplyd (No. 27) is marked with an asterisk.
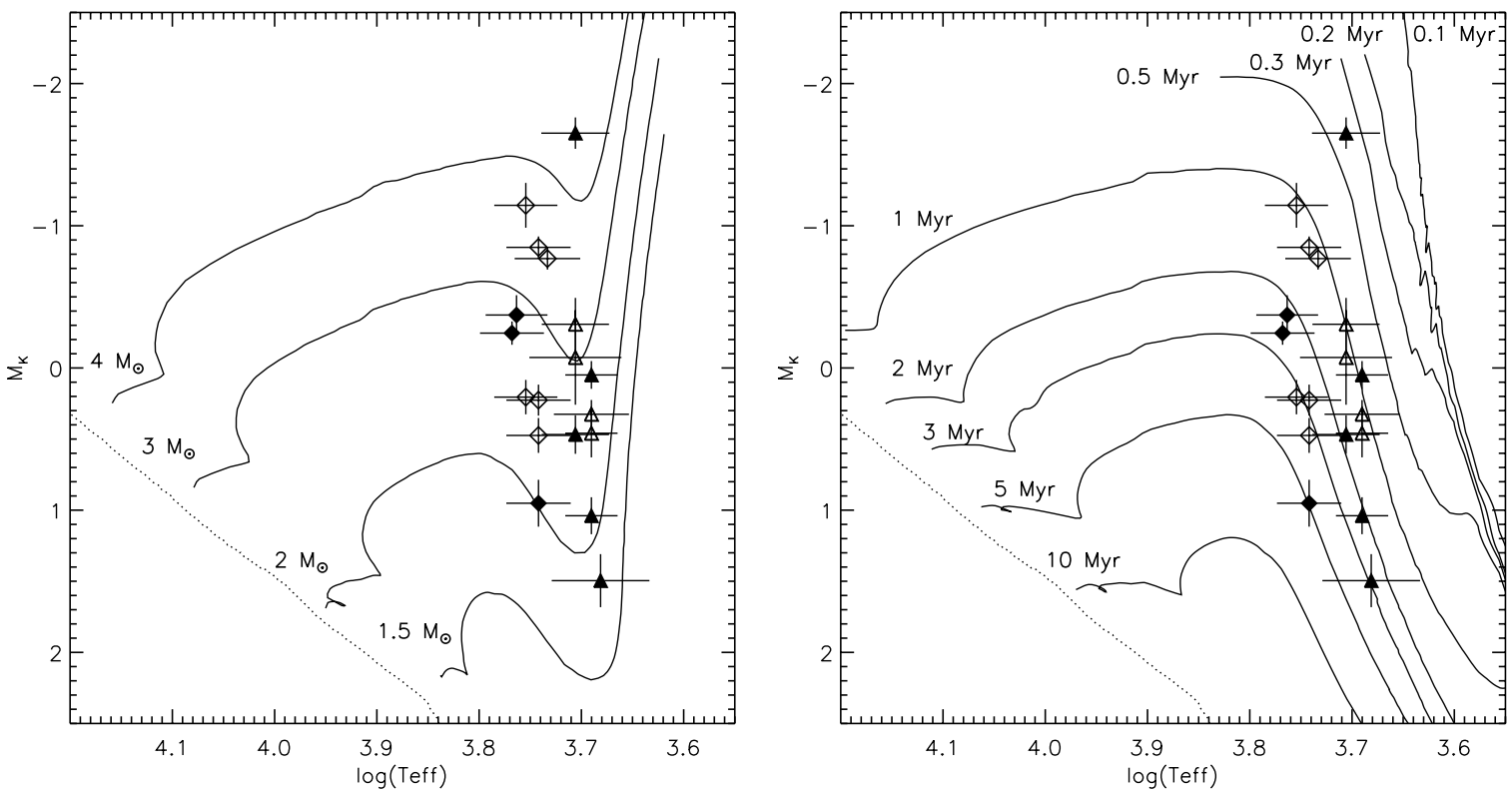

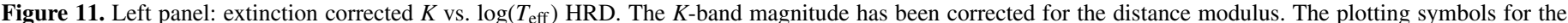

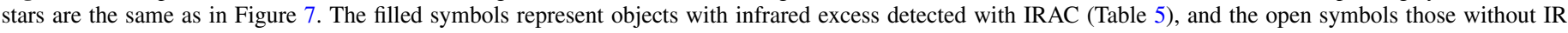

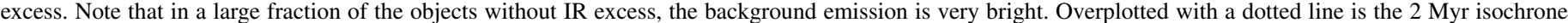

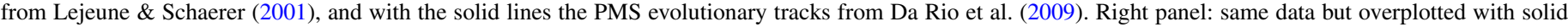
lines showing the isochrones from Da Rio et al. (2009).

5 Myr isochrones would have served equally well. A $20 M_{\odot}$ star does not evolve significantly off the main sequence for the first 8 Myr of its life (Lejeune \& Schaerer 2001), therefore we adopt 8 Myr as an upper limit for the age of RCW 34.

The solid lines in the left panel represent the evolutionary tracks taken from Da Rio et al. (2009) using the models of Siess et al. (2000) for four different masses; 4, 3, 2, and $1.5 M_{\odot}$. Comparison of the location of the PMS stars with these evolutionary tracks yields an approximate mass varying from $\sim 3 M_{\odot}$ for the brightest stars to $1.5 M_{\odot}$.

Overplotted in the right panel of Figure 11 are the PMS isochrones (solid lines) between 0.1 and $10 \mathrm{Myr}$. The location of the G- and $\mathrm{K}$ stars is consistent with a range of ages. The brightest object (No. 6) lies between the 0.3 and $0.5 \mathrm{Myr}$ isochrones; however, the majority of the objects span of the 1-3 Myr isochrones, with the more massive objects closer to the $1 \mathrm{Myr}$ isochrone. For the less massive objects, the spread in age is larger, most likely due to higher uncertainties in the spectral type determination. The location of the PMS stars suggests an age of $2 \pm 1$ Myr.

Comparison of the location of the PMS stars in the HRD with those of the Herbig AeBe stars (van den Ancker et al. 1998) shows that the PMS stars are younger than the Herbig AeBe stars and will evolve from their present G- and K-spectral type to late $\mathrm{B}, \mathrm{A}$, or early $\mathrm{F}$ spectral type when they become mainsequence stars. This also explains the fact that no A or F stars are spectroscopically detected, as they are still in the PMS phase at cooler temperatures. 
Even though the PMS stars are younger than the Herbig AeBe stars, they have, in contrast to the Herbig stars (e.g., Eiroa et al. $2001)$, no $K$-band excess. This could be the result of a different disk structure, but also caused by the fact that the PMS stars are emitting most of their energy in the near-infrared wavelength regime, where the SEDs of Herbig AeBe stars peak in the optical, thereby outshining the contribution of the circumstellar disk.

The extinction measurements show that the sources north of the HiI region are more deeply embedded in the molecular cloud. A younger age for this region is suggested by the presence of several protostars as well as outflows and a dense molecular cloud, which would suggest a younger age for the PMS object in this area as well. The PMS stars north of the HII region (No. 6, No. 8, No. 16, and No. 24) all have the brightest absolute magnitude in their temperature bin with source No. 6 as an extreme example. Their position in the HRD could suggest that they are the youngest of the stars of given spectral type. However, their high extinction leads to a selection effect (only the brightest sources are detected). Fainter sources would fall below the limit where SINFONI spectra could be classified. So, if there were an age difference, the current data would not be able to quantify this. Deeper spectroscopic observations to find more of the high-extinction PMS stars north of the $\mathrm{H}$ II region would be required to make any definitive statements.

\subsection{Deeply Embedded Population}

As the SINFONI observations show, large-extinction variations are present in RCW 34. The high extinction toward the north means that we miss sources that are too deeply embedded to be visible in $K$ band. In our IRAC catalog, we found two sources with no near-infrared counterpart (No. 28 and No. 29 in Table 6). Both sources have IRAC colors of deeply embedded protostars (class $0 / \mathrm{I}$ ).

Source No. 28 is located in the northeast corner of the SINFONI field, only $3^{\prime \prime}$ away from one of the outflows detected in the $\mathrm{H}_{2}$ line emission (outflow (c), Section 3.2.2). The other embedded source (No. 29) is located very close to source No. 1, but is not related. Thomas et al. (1973) and Williams et al. (1977) detected No. 29 as a mid-infrared excess toward vdBH 25a. The IRAC images show, however, that it is a separate source. The SED of No. 29 is difficult to determine; at $3.6 \mu \mathrm{m}$, the contribution of the $08.5 \mathrm{~V}$ star is still present, and at 5.8 and $8.0 \mu \mathrm{m}$, this object is the brightest source at MIR wavelengths. To determine a better SED, high-resolution and deeper mid-infrared observations are needed to discriminate between the emission from the protostar and the arc in the north. Additionally, as discussed above, the IRAC counterpart of source No. 14 could be another embedded protostar not seen at NIR wavelengths.

Outside the SINFONI field, several other protostars are located. Some of these objects seem to have a $K$-band counterpart in the SOFI image of Bik (2004), suggesting that the objects in our SINFONI field are even more embedded than those located a bit further away from the arc.

\subsection{Stellar Population of the Surroundings}

IRAC imaging reveals a large bubble surrounded by extended $8 \mu \mathrm{m}$ emission. Inside this bubble is a cluster of intermediateand low-mass class II objects. Typical ages for class II objects are of the order of a few megayears (Kenyon \& Hartmann 1995; White et al. 2007), not distinguishable from the age of the stars in the $\mathrm{H}$ II region.
The class 0/I stars, identified mainly close to the bright arc at the northern edge of the $\mathrm{H}$ II region, might be significantly younger. Whitney et al. (2003) suggest an age of $\sim 10^{5} \mathrm{yr}$ for class I sources; however, White et al. (2007) present evidence that the low-mass class I objects in Taurus gave a similar age as the class II sources. The location of the class $0 /$ I sources coincides with methanol masers, CS emission, high extinction and outflows, and therefore are most likely younger than the objects inside the bubble and the H II region.

Comparing the 2MASS $K$-band magnitudes of the class II stars in the bubble with the $K$-band magnitudes of the PMS stars in the $\mathrm{H}$ II region shows that 13 out of 17 class II objects are about a magnitude brighter. The other four are of similar magnitude. Additionally, in a $(J-H)$ versus $(H-K)$ color-color plot, most of the class II sources have a near-infrared excess and cover the same region in the color-color diagram as the Herbig Ae stars (Eiroa et al. 2001).

The difference in $K$-band magnitude could be explained with the presence of a $K$-band excess, suggesting that the class II sources are more similar to the Herbig Ae stars and are therefore more evolved than the PMS stars. An optical spectroscopic classification is needed to confirm this.

The location of the central star of the HII region, near the northern edge of the bubble suggests that this source might not have created the bubble. Also the $\mathrm{H} \alpha$ (Parker \& Phillipps 1998), as well as the $\mathrm{Br} \gamma$ emission, peaks at the location of the $\mathrm{O}$ star. In the rest of the bubble only faint $\mathrm{H} \alpha$ emission is present, indicating the absence of massive stars inside the bubble. Apart from the class II sources detected in the bubble, one source in the center (Figure 3) is detected without infrared excess. The magnitude at $3.6 \mu \mathrm{m}(10.0 \pm 0.02$ mag) is compatible with a B0V star at the distance of RCW 34. However, the optical photometry of the source taken from the USNO catalog (Monet 1998) is not compatible with a (reddened) early-type star. Therefore, we conclude that this star is an unrelated, foreground object. This raises the question whether other mechanisms could be responsible for the bubble. The scenario that the bubble would be created by a supernova seems unlikely. As the stars in the bubble are only a few megayears old, the supernova needs to come from an early-type $\mathrm{O}$ star. Assuming a normal IMF, the other $\mathrm{O}$ stars still would have to be visible.

Massive stars affect their environment by ionizing photons and stellar winds. Estimating the mechanical luminosity $\left(E_{\text {mech }}=1 / 2 \mathrm{Mv}^{2}\right)$ needed to blow a bubble with a radius of $2.5 \mathrm{pc}$ inside a medium with a typical molecular cloud density $\left(10^{3} \mathrm{~cm}^{-3}\right)$, expanding at $10 \mathrm{~km} \mathrm{~s}^{-1}$ would be on the order of $10^{42} \mathrm{erg} \mathrm{s}^{-1}$. As a comparison, the wind luminosity of a mid-B star is around $10^{30} \mathrm{erg} \mathrm{s}^{-1}$ (Tan 2004).

Assuming $\sim 10^{44}$ ionizing photons $\mathrm{s}^{-1}$, typical for a mid-B star and a density of $10^{3} \mathrm{~cm}^{-3}$, the Strömgren radius is $0.014 \mathrm{pc}$. This is a factor 100 lower than the observed radius of the bubble $(2.5 \mathrm{pc})$. Assuming that the 20 observed class II stars all emit the same number of ionizing photons and assuming a density of $100 \mathrm{~cm}^{-3}$, the Strömgren radius would be comparable to that of the measured bubble size.

Another possibility is that the OB star, which created the bubble, has been expelled from the center of the cluster due to a binary interaction, similar to what is suggested for the origin of $\mathrm{BN}$ in Orion (Rodríguez et al. 2005). The central star of the $\mathrm{H}$ II region could be one of the stars from the disrupted binary, with the other star moving in the opposite direction. With the current data we cannot exclude this scenario, detailed velocity 
measurements of the star in the $\mathrm{H}$ II region could give more insights into this scenario.

\section{DISCUSSION}

The interaction region between the $\mathrm{HII}$ region and the molecular cloud north of it raise the suggestion that some form of triggered star formation plays a role here. After having derived several properties of the $\mathrm{H}$ II region, the molecular cloud and the adjacent bubble we can now attempt to reconstruct the way this region has formed.

RCW 34 is composed of a large bubble in which intermediateand low-mass PMS stars have formed with no evidence for a central ionizing source. The ionizing source and its surrounding $\mathrm{H}$ II region, however, are located at the edge of the bubble, very close to the molecular cloud. The H II region and the molecular cloud are separated by an ionization front and a PDR traced by $\mathrm{H}_{2}$ emission and 5.8 and $8.0 \mu \mathrm{m}$ emission. The age of the stars inside the bubble (several Myr) is not known with sufficient accuracy to detect an age difference with the stars inside the $\mathrm{H}$ II region ( $2 \pm 1 \mathrm{Myr})$. The age of the objects forming inside the molecular cloud, however, is most likely younger, as several tracers of active star formation have been detected.

Inside the $\mathrm{H}$ II region, a north-south velocity gradient has been detected from $\mathrm{H} \alpha$ spectroscopy, resembling that of S88B, a proto-typical champagne flow (Garay et al. 1998; Stahler \& Palla 2005). The detection of the champagne flow, as well as the presence of the ionization front and PDR in the north, suggest that the $\mathrm{H}$ II region is formed at the edge of the molecular cloud. Due to the lower density in the bubble, the $\mathrm{H}$ II region can expand into the bubble creating the observed velocity gradient

We already can exclude one of the triggered star formation scenarios. The collect and collapse scenario, where the starforming clumps are formed by swept-up material is very likely not the scenario that plays a role here. Firstly, only at the northern edge of the bubble is a molecular clump present, while more clumps around the bubble are expected (Deharveng et al. 2005). Secondly, it is very unlikely that the energy output of a cluster of only intermediate stars would be enough to sweep up a dense molecular clump of more than $1000 M_{\odot}$ as detected in CS emission.

Also unlikely is that the formation of the $\mathrm{H}$ II region and the $\mathrm{O}$ star caused both the bubble and the star formation in the Molecular Cloud. The difference in evolutionary stage (class II stars in the bubble and class 0/I stars in the molecular cloud) is difficult to explain by the triggering due to the $\mathrm{H}$ in region alone.

The location of the bubble, $\mathrm{H}$ II region, and molecular cloud suggest a sequence of events. First, the cluster of stars formed which created the bubble. After that, the H II region formed at the border of the bubble and the larger molecular cloud; the latter currently forming stars. The location of the H II region with respect to the molecular cloud as well as the presence of the PDR shows that the $\mathrm{O}$ star is influencing the molecular cloud and probably also inducing the current star formation.

The question remains whether the formation of the bubble triggered the other star formation events. A mechanism whereby the energy output of the stars inside the bubble would be pushing a pre-existing core to collapse could be at work here. If a molecular core present at the edge of the molecular cloud were in equilibrium, a small push from the bubble could have made the core collapse and enable the formation of the OB stars.

Another alternative would be that the triggering mechanism is external and that a wave of star formation is propagating through the molecular cloud resulting in the formation of the different sites of star formation.

To determine which scenario is the correct one, a better age determination of the class II sources by means of optical or nearinfrared spectroscopy would be needed. A triggering scenario would result in an age difference between the stars in the bubble and the $\mathrm{H}$ II region.

\section{CONCLUSIONS}

In this paper, we have presented SINFONI integral field spectroscopy of the H II region RCW 34 as well as Spitzer/IRAC imaging of the surrounding area. The results can be summarized as follows.

1. RCW 34 consists of three separate regions, a large bubble outlined by Spitzer/IRAC emission with an H II region at the northern edge of the bubble and a dense molecular cloud just north of the $\mathrm{H}$ II region. IRAC photometry of the sources inside the bubble reveals a cluster of intermediate class II sources and shows that no OB star is located near the center of the bubble. The class $0 / \mathrm{I}$ objects are mostly detected toward the dense molecular cloud in the north, suggesting active star formation present in the molecular cloud.

2. SINFONI spectroscopy of the H II region reveals an extinction gradient from north $\left(A_{V}=15.9 \mathrm{mag}\right)$ to south ( $\left.A_{V}=1.5 \mathrm{mag}\right)$, supportive of the champagne flow hypothesis measured in the $\mathrm{H} \alpha$ velocity profile. The region between the $\mathrm{H}$ in region and the molecular cloud is identified as a PDR, where the $\mathrm{H}_{2}$ emission originates from UV fluorescence. Additionally, two outflows are identified based on their $\mathrm{H}_{2}$ emission.

3. SINFONI spectroscopy identifies the three brightest stars as ionizing sources of the $\mathrm{H}$ II region. The main ionizing source, No. 1, is of spectral type $08.5 \mathrm{~V}$ while the two other stars are $\mathrm{B} 0.5 \mathrm{~V}$ and $\mathrm{B} 3 \mathrm{~V}$ stars. This results in a revised distance estimate of $2.5 \pm 0.2 \mathrm{kpc}$ for RCW 34 . Additionally, we detected $21 \mathrm{G}$ and $\mathrm{K}$ stars of which two stars are foreground objects and 19 objects are PMS cluster members. With a mass between 2 and $3 M_{\odot}$, these objects are still contracting to the main sequence and are the precursors of the $\mathrm{A}$ and $\mathrm{F}$ stars. Combining the location of those objects in an HRD with PMS evolutionary models results in an age estimate of $2 \pm 1$ Myr for RCW 34 .

4. In this scenario, the cluster stars created the bubble, which then collapsed a pre-existing dense core at the edge of the molecular cloud. The resulting $\mathrm{H}$ II region would encounter the density gradient at the edge of the cloud, and thus develop as a champagne flow. Whether the bubble was the original trigger, or if there was some other, external trigger, cannot be answered with the current data; a detailed spectroscopic study of the bubble stars will be required.

This study shows that the interaction between the young massive stars and the surrounding molecular cloud could not only lead to the destruction of the cloud, but also to the formation of a younger generation of stars. In the majority of our regions, we have identified multiple generations of star formation. Several papers are in preparation to discuss the remainder of the sample of 10 clusters observed with SINFONI (e.g., Y. Wang et al. 2010, in preparation; T. Vasyunina et al. 2010, in preparation) and more final conclusions will be presented as a final paper discussing the survey. 
The authors thank F. Eisenhauer for providing the data reduction software SPRED, A. Modigliani for help in the data reduction, R. Davies for providing his routines to remove the $\mathrm{OH}$ line residuals and N. Da Rio for providing the isochrones and evolutionary tracks, as well as L. Decin for discussions on the classification of the PMS stars. The authors thank the Paranal staff for carrying out the observations.

E.P. is funded by the Spanish MEC under the ConsoliderIngenio 2010 Program grant CSD2006-00070: First Science with the GTC, and under grants AYA2007-67456-C02-02/ AYA2008-06166-C03-02. H.B. and A.S. acknowledge financial support by the Emmy Noether Program of the Deutsche Forschungsgemeinschaft (DFG; grants BE2578, STO 496/3). M.B.N.K. was supported by the Peter and Patricia Gruber Foundation and by PPARC/STFC under grant number $\mathrm{PP} / \mathrm{D} 002036 / 1$. This work is based on observations made with the Spitzer Space Telescope, which is operated by the Jet Propulsion Laboratory, California Institute of Technology under a contract with the National Aeronautics and Space Administration (NASA). NSO/Kitt Peak FTS data used here were produced by NSF/NOAO.

Facilities: VLT:Yepun (SINFONI), Spitzer (IRAC)

\section{REFERENCES}

Abuter, R., Schreiber, J., Eisenhauer, F., Ott, T., Horrobin, M., \& Gillesen, S. 2006, New Astron. Rev, 50, 398

Allen, L. E., et al. 2004, ApJS, 154, 363

Aller, L. H., et al. 1982, Landolt-Börnstein: Numerical Data and Functional Relationships in Science and Technology (Berlin: Springer)

Alvarez, C., Feldt, M., Henning, T., Puga, E., Brandner, W., \& Stecklum, B. 2004, ApJS, 155, 123

Bik, A. 2004, PhD thesis, Univ. of Amsterdam

Bik, A., Kaper, L., Hanson, M. M., \& Smits, M. 2005, A\&A, 440, 121

Blum, R. D., Conti, P. S., \& Damineli, A. 2000, AJ, 119, 1860

Blum, R. D., Ramond, T. M., Conti, P. S., Figer, D. F., \& Sellgren, K. 1997, AJ, 113,1855

Bonnet, H., et al. 2004, Messenger, 117, 17

Braz, M. A., \& Epchtein, N. 1983, A\&A, 54, 167

Bronfman, L., Nyman, L.-A., \& May, J. 1996, A\&AS, 115, 81

Cardelli, J. A., Clayton, G. C., \& Mathis, J. S. 1989, ApJ, 345, 245

Caswell, J. L., Batchelor, R. A., Forster, J. R., \& Wellington, K. J. 1989, Aust. J. Phys., 42, 331

Caswell, J. L., \& Haynes, R. F. 1987, A\&A, 171, 261

Chan, S. J., Henning, T., \& Schreyer, K. 1996, A\&AS, 115, 285

Churchwell, E. B., et al. 2006, ApJ, 649, 759

Cohen, M., \& Kuhi, L. V. 1979, ApJS, 41, 743

Cohen, M., Wheaton, W. A., \& Megeath, S. T. 2003, AJ, 126, 1090

Cushing, M. C., Rayner, J. T., \& Vacca, W. D. 2005, ApJ, 623, 1115

Da Rio, N., Gouliermis, D. A., \& Henning, T. 2009, ApJ, 696, 528

Davies, R. I. 2007, MNRAS, 375, 1099

Davis, C. J., Smith, M. D., Stern, L., Kerr, T. H., \& Chiar, J. E. 2003, MNRAS, 344,262

Deharveng, L., Zavagno, A., \& Caplan, J. 2005, A\&A, 433, 565

DeRose, K. L., Bourke, T. L., Gutermuth, R. A., Wolk, S. J., Megeath, S. T., Alves, J., \& Nürnberger, D. 2009, AJ, 138, 33

Eiroa, C., et al. 2001, A\&A, 365, 110

Eisenhauer, F., et al. 2003, Proc. SPIE, 4841, 1548

Elmegreen, B. G. 1998, in ASP Conf. Ser. 148, Origins, ed. C. E. Woodward, J. M. Shull, \& H. A. Thronson, Jr. (San Francisco, CA: ASP), 150

Elmegreen, B. G., \& Lada, C. J. 1977, ApJ, 214, 725

Fazio, G. G., et al. 2004, ApJS, 154, 10

Feldt, M., et al. 2003, ApJ, 599, L91

Garay, G., Lizano, S., Gomez, Y., \& Brown, R. L. 1998, ApJ, 501, 710

Gum, C. S. 1955, MNRAS, 67, 155
Gyulbudaghian, A. L.. \& May, J. 2007, Astrophys., 50, 1

Hanson, M. M., Conti, P. S., \& Rieke, M. J. 1996, ApJS, 107, 281

Hanson, M. M., Howarth, I. D., \& Conti, P. S. 1997, ApJ, 489, 698

Hanson, M. M., Luhman, K., \& Rieke, G. H. 2002, ApJS, 138, 35

Hanson, M. M., Rieke, G. H., \& Luhman, K. L. 1998, AJ, 116, 1915

Herbst, W. 1975, AJ, 80, 683

Heydari-Malayeri, M. 1988, A\&A, 202, 240

Hillenbrand, L. A. 1997, AJ, 113, 1733

Hollenbach, D., \& McKee, C. F. 1989, ApJ, 342, 306

Kendall, T. R., de Wit, W.-J., \& Yun, J. L. 2003, A\&A, 408, 313

Kenyon, S. J., \& Hartmann, L. 1995, ApJS, 101, 117

Koornneef, J. 1983, A\&A, 128, 84

Kurucz, R. L. 1993, Kurucz CD-ROM No. 13 (Cambridge, MA: Smithsonian Astrophysical Obs.)

Lejeune, T., \& Schaerer, D. 2001, A\&A, 366, 538

Liseau, R., Lorenzetti, D., Nisini, B., Spinoglio, L., \& Moneti, A. 1992, A\&A, 265,577

Luhman, K. L. 1999, ApJ, 525, 466

Lumsden, S. L., Puxley, P. J., Hoare, M. G., Moore, T. J. T., \& Ridge, N. A. 2003, MNRAS, 340, 799

Makovoz, D., Khan, I., \& Masci, F. 2006, Proc. SPIE, 6065, 330

Martin-Hernández, N. L., Bik, A., Puga, E., Nuernberger, D. E. A., \& Bronfman, L. 2008, A\&A, 489, 229

Martins, F., \& Plez, B. 2006, A\&A, 457, 637

Martins, F., Schaerer, D., \& Hillier, D. J. 2002, A\&A, 382, 999

Martins, F., Schaerer, D., \& Hillier, D. J. 2005, A\&A, 436, 1049

Megeath, S. T., et al. 2004, ApJS, 154, 367

Monet, D. 1998, USNO-A2.0 Catalog of Astrometric Standards (Washington, DC: US Naval Obs.)

Murphy, D. C., \& May, J. 1991, A\&A, 247, 202

Muzerolle, J., Hartmann, L., \& Calvet, N. 1998, AJ, 116, 2965

O'Dell, C. 1998, AJ, 115, 263

O'Dell, C. 2001, ARA\&A, 39, 99

Parker, Q. A., \& Phillipps, S. 1998, Publ. Astron. Soc. Aust., 15, 28

Puga, E., Feldt, M., Alvarez, C., Henning, T., Apai, D., Coarer, E. L., Chalabaev, A., \& Stecklum, B. 2006, ApJ, 641, 373

Rayner, J. T., Cushing, M. C., \& Vacca, W. D. 2009, ApJS, 185, 289

Rodgers, A. W., Campbell, C. T., \& Whiteoak, J. B. 1960, MNRAS, 121, 103

Rodríguez, L. F., Poveda, A., Lizano, S., \& Allen, C. 2005, ApJ, 627, L65

Rost, S., Eckart, A., \& Ott, T. 2008, A\&A, 485, 107

Schreiber, J., Thatte, N., Eisenhauer, F., Tecza, M., Abuter, R., \& Horrobin, M. 2004, in ASP Conf. Ser. 314, Astronomical Data Analysis Software and Systems XIII, ed. F. Ochsenbein, M. G. Allen, \& D. Egret (San Francisco, CA: ASP), 380

Siess, L., Dufour, E., \& Forestini, M. 2000, A\&A, 358, 593

Stahler, S. W., \& Palla, F. 2005, in The Formation of Stars, ed. S. W. Stahler \& F. Palla (New York: Wiley), 865

Stecklum, B., Henning, T., Feldt, M., Hayward, T. L., Hoare, M. G., Hofner, P., \& Richter, S. 1998, AJ, 115, 767

Storey, P. J., \& Hummer, D. G. 1995, MNRAS, 272, 41

Tan, J. C. 2004, ApJ, 607, L47

Thomas, J. A., Hyland, A. R., \& Robinson, G. 1973, MNRAS, 165, 201

Turner, J., Kirby-Docken, K., \& Dalgarno, A. 1977, ApJS, 35, 281

van den Ancker, M. E., de Winter, D., \& Djie, H. R. E. T. A. 1998, A\&A, 330, 145

van den Bergh, S., \& Herbst, W. 1975, AJ, 80, 208

Walsh, A. J., Hyland, A. R., Robinson, G., \& Burton, M. G. 1997, MNRAS, 291,261

Watson, A. M., \& Hanson, M. M. 1997, ApJ, 490, L165

White, R. J., Greene, T. P., Doppmann, G. W., Covey, K. R., \& Hillenbrand, L. A. 2007, in Protostars and Planets V, ed. B. Reipurth, D. Jewitt, \& K. Keil (Tucson, AZ: Univ. Arizona Press), 117

Whitney, B. A., Wood, K., Bjorkman, J. E., \& Cohen, M. 2003, ApJ, 598, 1079

Williams, P. M., Beattie, D. H., \& Stewart, J. M. 1977, MNRAS, 178, 619

Winston, E., et al. 2009, AJ, 137, 4777

Yamaguchi, N., Mizuno, N., Saito, H., Matsunaga, K., Mizuno, A., Ogawa, H., \& Fukui, Y. 1999, PASJ, 51, 775

Zhang, Q., Ho, P. T. P., \& Ohashi, N. 1998, ApJ, 494, 636

Zinchenko, I., Mattila, K., \& Toriseva, M. 1995, A\&AS, 111, 95

Zinnecker, H., \& Yorke, H. W. 2007, ARA\&A, 45, 481 